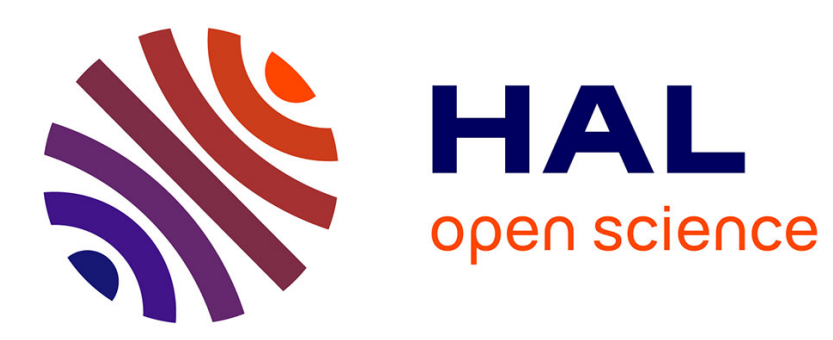

\title{
Multivariate utility maximization with proportional transaction costs and random endowment
}

Giuseppe Benedetti, Luciano Campi

\section{To cite this version:}

Giuseppe Benedetti, Luciano Campi. Multivariate utility maximization with proportional transaction costs and random endowment. 2011. hal-00586377

\section{HAL Id: hal-00586377 \\ https://hal.science/hal-00586377}

Preprint submitted on 15 Apr 2011

HAL is a multi-disciplinary open access archive for the deposit and dissemination of scientific research documents, whether they are published or not. The documents may come from teaching and research institutions in France or abroad, or from public or private research centers.
L'archive ouverte pluridisciplinaire HAL, est destinée au dépôt et à la diffusion de documents scientifiques de niveau recherche, publiés ou non, émanant des établissements d'enseignement et de recherche français ou étrangers, des laboratoires publics ou privés. 


\title{
Multivariate utility maximization with proportional transaction costs and random endowment
}

\author{
Giuseppe Benedetti* Luciano Campi ${ }^{\dagger}$
}

April 15, 2011

\begin{abstract}
In this paper we deal with a utility maximization problem at finite horizon on a continuous-time market with conical (and time varying) constraints (particularly suited to model a currency market with proportional transaction costs). In particular, we extend the results in [CO10] to the situation where the agent is initially endowed with a random and possibly unbounded quantity of assets. We start by studying some basic properties of the value function (which is now defined on a space of random variables), then we dualize the problem following some convex analysis techniques which have proven very useful in this field of research. We finally prove the existence of a solution to the dual and (under an additional boundedness assumption on the endowment) to the primal problem. The last section of the paper is devoted to an application of our results to utility indifference pricing.
\end{abstract}

Key-words: Transaction costs, foreign exchange market, multivariate utility function, optimal portfolio, duality theory, random endowment, utility-based pricing.

JEL Classification: G11

AMS Classification (2000): Primary - 91B28, Secondary - 49N15, 49J40, 49J55.

\section{Introduction}

We place ourselves in the framework of a continuous-time market with proportional transaction costs as described in [CO10] and in [CS06]. The agent's objective is to maximize his utility at a fixed terminal date $T$ by trading in the available assets. The model is very general, as it allows the portfolio process to be driven by any cone-valued process, provided it satisfies some regularity assumptions. In the most common version of the model, the cones are generated by the evolution of bid-ask prices (which may possibly have jumps) and therefore they describe market frictions due to transaction costs. Also in this framework, the model preserves a great generality as the modeling of bid-ask prices does not pass through asset prices and transaction costs dynamics separately. This approach, based on the key concept of solvency cones, was first introduced in [Kab99] and it has been further developed by many authors in the last decade (for more details, see the recent book [KS09] and the references therein).

The agent's preferences are described by a multivariate utility function (see Section 2.2) supported on $\mathbb{R}_{+}^{d}$, reflecting the idea that the agent will not necessarily liquidate his positions to a single numeraire at the final date (which is realistic, in particular, on a currency market). We also make the following assumptions.

Assumption 1.1 The utility function $U: \mathbb{R}^{d} \rightarrow[-\infty, \infty)$ satisfies the following conditions:

(i) $U$ is measurable;

(ii) $U$ is strictly concave on the interior of $\mathbb{R}_{+}^{d}$;

(iii) $U$ is essentially smooth and its gradient diverges at the boundary of $\mathbb{R}_{+}^{d}$ (see Definition 2.5);

(iv) $U$ is asymptotically satiable (see Definition 2.6).

${ }^{*}$ CREST (Finance/Insurance Laboratory) and University Paris-Dauphine

${ }^{\dagger}$ CEREMADE, University Paris-Dauphine.

The second author thanks the "Chair Les Particuliers Face aux Risques", Fondation du Risque (Groupama-ENSAEDauphine) and the "Chair Finance and Sustainable Development" sponsored by EDF for their support. 
As in [Kam01], the utility function is then extended to $D>d$ assets in order to model the investor's preferences towards a restricted set of assets in a larger economy. This is motivated by the fact that the agent may be ultimately interested in consuming a small set of assets at the date $T$, but he will trade in all available assets in order to reach his objective). Hence we define $\tilde{U}: \mathbb{R}^{D} \rightarrow[-\infty,+\infty)$ by

$$
\tilde{U}(x)= \begin{cases}U\left(x_{1}, \ldots, x_{d}\right), & \text { if } x \in \mathbb{R}_{+}^{D} \\ -\infty & \text { otherwise }\end{cases}
$$

In the formulation of [CO10] the investor is initially endowed with a deterministic amount $x \in \mathbb{R}^{D}$ of different assets, while in this paper we extend those results by assuming that the initial endowment is a random variable, that we call $\mathcal{E}:=\left(\mathcal{E}_{1}, \ldots, \mathcal{E}_{D}\right)$. For example the agent may have no assets at the beginning but he may have access to some contingent claims on these assets (such as a right to buy or to sell some of them at a future date).

The first systematic study of a utility maximization problem with a (bounded) random endowment in a general frictionless semimartingale model is due to [CSW01], where the authors considered univariate utility functions and used the duality approach based on some ideas already developed in [KrS99]. Important contributions in the same direction have later been given, among others, in [HG04] and [OZ09], where the boundedness condition on the endowment is relaxed and replaced by weaker requirements (those in [OZ09], in particular, have inspired the ones which are employed in this paper).

Duality methods in a utility maximization problem with transaction costs had been introduced for the first time in [CK96] in a diffusion market model with one risky asset, constant proportional transaction costs and no random endowment (for a more complete story we refer to the Introduction in [CO10]). [Bou02] investigated an optimization problem for an agent with a bounded random endowment, using the already mentioned idea of solvency cones introduced by Kabanov in a series of papers (see [KS09] for a reference). This new modeling approach paved the way for the more general model in [CS06] (with time varying and random proportional transaction costs), which in turn provided the necessary tools for the results in [CO10], where multivariate utility functions are introduced in the optimization problem (with deterministic endowment). We recall the paper [DPT01], where the topic of multivariate utility maximization has been studied for the first time (in a constant transaction cost framework).

In the present paper we extend the results in [CO10] to the case of an agent equipped with a possibly unbounded random endowment in a model where transaction costs are proportional, they can be random and have jumps. Moreover, we will use our duality results to obtain some general results on utility indifference pricing.

The subject of utility-based pricing of contingent claims has been an active (and quite natural) area of research since the introduction and development of incomplete market models, in which the replication paradigm is no longer sufficient to find a unique price (hence utility comes in as an additional criterion of choice). The idea of utility indifference pricing has been first introduced in a dynamic hedging framework by [HN89] and it has been further extended by other authors in different settings, possibly under different names, see for example [Mu99] and [OZ09] (which is our main reference). In fact, the underlying concept of certainty equivalent is quite pervasive in the whole economics literature, because of its natural and intuitive interpretation. We refer to [HH09] for a more detailed overview on this subject.

Before proceeding, Section 2 will give some details on the transaction cost model we work on, as well as some preliminaries on the main mathematical tools that we are going to employ. The main results on duality and existence of an optimizer are presented in Section 3, while in Section 4 we propose an application to utility-based pricing of contingent claims.

\section{Preliminaries}

In this section we present all the preliminary concepts and notation which are required for the analysis of the optimization problem.

\subsection{Cones and transaction costs}

A general and convenient description of a large class or market constraints and/or frictions can be provided by a Kabanov-type market model, which is centered on the idea of cone-valued processes (evolving in continuous time in our framework). Let $\left(\Omega,\left(\mathscr{F}_{t}\right)_{t \in[0, T]}, \mathbb{P}\right)$ be a filtered probability space satisfying the usual conditions and supporting all processes appearing in this paper. We will use the 
notation $\chi_{A}$ for the indicator function of a set $A$ and cone $(A)$ to denote the cone generated by any set $A$ in $\mathbb{R}^{D}$.

A $\mathcal{C}$-valued process is defined as a sequence of set-valued mappings $K=\left(K_{t}\right)_{t \in[0, T]}$ specified by a countable sequence of adapted $\mathbb{R}^{D}$-valued processes $X^{n}=\left(X_{t}^{n}\right)$ such that, for all $t$ and $\omega$, only a finite but nonzero number of $X_{t}(\omega)$ is different from zero and

$$
K_{t}(\omega):=\operatorname{cone}\left\{X_{t}^{n}(\omega), n \in N\right\}
$$

which implies that $K_{t}(\omega)$ is a polyhedral cone (by the so-called Farkas-Minkowski-Weyl Theorem, see e.g. Section 5.1 in the Appendix in [KS09]). The cones $K_{t-}$ are the ones generated by the left limits of the generators. As we shall see in a moment, these cones are there to describe the trading possibilities of an investor over time, i.e. to model the evolution of the portfolio processes.

Let $K_{s, t}(\omega)$ denote the closure of cone $\left\{K_{r}(\omega), s \leq r<t\right\}$, and let

$$
K_{s, t+}(\omega):=\bigcap_{\epsilon>0} K_{s, t+\epsilon}(\omega), K_{s-, t}(\omega):=\bigcap_{\epsilon>0} K_{s-\epsilon, t}(\omega) .
$$

In order to derive useful results one needs some regularity assumptions that we list here. Recall that a cone $K$ is proper if $K \cap(-K)=\{0\}$.

Assumption $2.1 \quad$ (i) The cones $K_{t}$ and $K_{t-}$ are proper and contain $\mathbb{R}_{+}^{D}$ (Efficient friction)

(ii) $K_{t, t+}=K_{t}, K_{t-, t}=K_{t-}$ and $K_{t-, t+}=\operatorname{cone}\left\{K_{t-}, K_{t}\right\}$ for all $t$.

REMARK 2.1 It can be shown (see [KS09], p.165) that (ii) is verified if (i) is true and all cones $K_{t}$ and $K_{t-}$ can be generated by a finite number of càdlàg vector processes.

EXAMPLE 2.1 Even though all the results of this paper are true just under the above assumptions, we give here an example of how cone processes can be constructed in a particular (but still quite general) model of a market with transaction costs, which is the main situation we have in mind (and which justifies the title of the paper). In such a model, formalized in [CS06] (see also [S04]), all agents can trade in $D$ assets according to a random and time varying bid-ask matrix. A $D \times D$ matrix $\Pi=\left(\pi^{i j}\right)_{1 \leq i, j \leq D}$ is called a bid-ask matrix if (i) $\pi^{i j}>0$ for every $1 \leq i, j \leq D$, (ii) $\pi^{i i}=1$ for every $1 \leq i \leq D$, and (iii) $\pi^{i j} \leq \pi^{i k} \pi^{k j}$ for every $1 \leq i, j, k \leq D$.

Given a bid-ask matrix $\Pi$, the solvency cone $K(\Pi)$ is defined as the convex polyhedral cone in $\mathbb{R}^{D}$ spanned by the canonical basis vectors $e^{i}, 1 \leq i \leq D$ of $\mathbb{R}^{D}$, and the vectors $\pi^{i j} e^{i}-e^{j}, 1 \leq i, j \leq D$. The convex cone $-K(\Pi)$ should be interpreted as those portfolios available at price zero.

We must now introduce randomness and time in the model. An adapted, càdlàg process $\left(\Pi_{t}\right)_{t \in[0, T]}$ taking values in the set of bid-ask matrices will be called a bid-ask process. Once a bid-ask process $\left(\Pi_{t}\right)_{t \in[0, T]}$ has been fixed, one can drop it from the notation by writing $K_{\tau}$ instead of $K\left(\Pi_{\tau}\right)$ for a stopping time $\tau$, coherently with the framework introduced above. Under the hypothesis of efficient friction (i), part (ii) of Assumption 2.1 is automatically satisfied in this case by Remark 2.1.

In accordance with the framework developed in [CS06] we make the following technical assumption throughout the paper. The assumption is equivalent to disallowing a final trade at time $T$, but it can be relaxed via a slight modification of the model (see [CS06, Remark 4.2]). For this reason, we shall not explicitly mention the assumption anywhere.

Assumption $2.2 \mathscr{F}_{T-}=\mathscr{F}_{T}$ and $\Pi_{T-}=\Pi_{T}$ a.s.

Given a cone $K$ in $\mathbb{R}^{D}$, its (positive) polar cone is defined by

$$
K^{*}=\left\{w \in \mathbb{R}^{D}:\langle v, w\rangle \geq 0, \forall v \in K\right\} .
$$

Definition 2.1 An adapted, $\mathbb{R}_{+}^{D} \backslash\{0\}$-valued, càdlàg martingale $Z=\left(Z_{t}\right)_{t \in[0, T]}$ is called a consistent price process for the $\mathcal{C}$-valued process $K$ if $Z_{t} \in K_{t}^{*}$ a.s. for every $t \in[0, T]$. Moreover, $Z$ will be called a strictly consistent price process if $Z_{t} \in \operatorname{int}\left(K_{t}^{*}\right)$ and $Z_{t-} \in \operatorname{int}\left(K_{t-}^{*}\right)$ a.s. for every $t \in[0, T]$. The set of all (strictly) consistent price processes will be denoted by $\mathcal{Z}\left(\mathcal{Z}^{s}\right)$.

The following assumption, which is used extensively in [CS06], will also hold throughout the paper. 
Assumption 2.3 (SCPS) Existence of a strictly consistent price system: $\mathcal{Z}^{s} \neq \emptyset$.

This assumption is intimately related to the absence of arbitrage (see also [JK95, GRS10, GK10, DGR11]).

Definition 2.2 Suppose that $K=\left(K_{t}\right)_{t \in[0, T]}$ is a $\mathcal{C}$-valued process such that Assumption 2.3 holds true. An $\mathbb{R}^{D}$-valued process $V=\left(V_{t}\right)_{t \in[0, T]}$ is called a self-financing portfolio process for the process $K$ if it satisfies the following properties:

(i) It is predictable and a.e. path has finite variation (not necessarily right-continuous).

(ii) For every pair of stopping times $0 \leq \sigma \leq \tau \leq T$, we have

$$
V_{\tau}-V_{\sigma} \in-K_{\sigma, \tau}
$$

A self-financing portfolio process $V$ is called admissible if it satisfies the additional property

(iii) There is a constant $a>0$ such that $V_{T}+a \mathbf{1} \in K_{T}$ a.s. and $\left\langle V_{\tau}+a \mathbf{1}, Z_{\tau}^{s}\right\rangle \geq 0$ a.s. for all $[0, T]$ valued stopping times $\tau$ and for every strictly consistent price process $Z^{s} \in \mathcal{Z}^{s}$. Here, $\mathbf{1} \in \mathbb{R}^{D}$ denotes the vector whose entries are all equal to 1.

Let $\mathcal{A}^{x}$ denote the set of all admissible, self-financing portfolio processes with initial endowment $x \in \mathbb{R}^{D}$, and let

$$
\mathcal{A}_{T}^{x}:=\left\{V_{T}: V \in \mathcal{A}^{x}\right\}
$$

be the set of all contingent claims attainable at time $T$ with initial endowment $x$. Note that $\mathcal{A}_{T}^{x}=x+\mathcal{A}_{T}^{0}$ for all $x \in \mathbb{R}^{D}$.

For the convenience of the reader we present a reformulation of [CS06, Theorem 4.1].

Theorem 2.1 (Super-Replication) Let $x \in \mathbb{R}^{D}$ and let $X$ be an $\mathscr{F}_{T}$-measurable, $\mathbb{R}_{+}^{D}$-valued random variable. Under Assumption 2.3 we have

$$
X \in \mathcal{A}_{T}^{x} \quad \text { if and only if } \quad \mathbb{E}\left[\left\langle X, Z_{T}^{s}\right\rangle\right] \leq\left\langle x, Z_{0}^{s}\right\rangle \text { for all } Z^{s} \in \mathcal{Z}^{s} .
$$

This result will be used in particular in the proof of Theorem 3.2 to show that our candidate for the optimizer in the utility maximization problem (with random endowment) is indeed an attainable contingent claim, i.e. the terminal value of an admissible portfolio.

\subsection{Convex analysis and utility functions}

The material of this section is mostly taken from Sections 2.2 and 2.3 in [CO10], where all the proofs can be found. We report here those results that we are going to use in our proofs for reader's convenience.

Let $(\mathscr{X}, \tau)$ be a locally convex topological vector space, and let $\mathscr{X}^{*}$ denote its dual space. Given a set $S \subseteq \mathscr{X}$ we let $\operatorname{cl}(S), \operatorname{int}(S), \operatorname{ri}(S)$ and aff $(S)$ denote respectively the closure, interior, relative interior and affine hull of $S$. We shall say that a set $C \subseteq \mathscr{X}$ is a convex cone if $\lambda C+\mu C \subseteq C$ for all $\lambda, \mu \geq 0$. Given set $S \subseteq \mathscr{X}$, we denote its polar cone by

$$
S^{*}:=\left\{x^{*} \in \mathscr{X}^{*}:\left\langle x, x^{*}\right\rangle \geq 0 \forall x \in S\right\} .
$$

Note that $S^{*}$ is weak* closed. A convex cone $C \subseteq \mathscr{X}$ induces a preorder $\succeq_{C}$ on $\mathscr{X}$ : We say that $x, x^{\prime} \in \mathscr{X}$ satisfy $x^{\prime} \succeq_{C} x$ if and only if $x^{\prime}-x \in C$. When we do not specify the cone in the notation, we always mean that it is $\mathbb{R}_{+}^{D}$.

Definition 2.3 (Dual functionals) (i) If $\mathfrak{U}: \mathscr{X} \rightarrow[-\infty, \infty$ ) is proper concave then we define its dual functional $\mathfrak{U}^{*}: \mathscr{X}^{*} \rightarrow(-\infty, \infty]$ by

$$
\mathfrak{U}^{*}\left(x^{*}\right):=\sup _{x \in \mathscr{X}}\left\{\mathfrak{U}(x)-\left\langle x, x^{*}\right\rangle\right\} .
$$

The dual functional $\mathfrak{U}^{*}$ is a weak ${ }^{*}$ lower semi-continuous, proper convex functional on $\mathscr{X}^{*}$. Note that $\mathfrak{U}^{*}=(\operatorname{cl}(\mathfrak{U}))^{*}($ see e.g. [Z02, Theorem 2.3.1]). 
(ii) If $\mathfrak{V}: \mathscr{X}^{*} \rightarrow(-\infty, \infty]$ is proper convex then we define the pre-dual functional ${ }^{*} \mathfrak{V}: \mathscr{X} \rightarrow[-\infty, \infty)$ by

$$
{ }^{*} \mathfrak{V}(x):=\inf _{x^{*} \in \mathscr{X}^{*}}\left\{\mathfrak{V}\left(x^{*}\right)+\left\langle x, x^{*}\right\rangle\right\} .
$$

Similarly, ${ }^{*} \mathfrak{V}$ is a weakly ${ }^{\dagger}$ upper semi-continuous, proper concave functional.

We say that $\mathfrak{U}$ is increasing with respect to a preorder $\succeq$ on $\mathscr{X}$, if $\mathfrak{U}\left(x^{\prime}\right) \geq \mathfrak{U}(x)$ for all $x, x^{\prime} \in \mathscr{X}$ such that $x^{\prime} \succeq x$.

Lemma 2.1 [CO10, Lemma 2.8] Let $\mathfrak{U}: \mathscr{X} \rightarrow[-\infty, \infty)$ be proper concave. Then $\mathfrak{U}^{*}$ is decreasing with respect to the preorder induced by $(\operatorname{dom}(\mathfrak{U}))^{*}$. Suppose furthermore that $\mathfrak{U}$ is increasing with respect to the preorder induced by some cone $C$. Then $\operatorname{dom}\left(\mathfrak{U}^{*}\right) \subseteq C^{*}$.

Definition 2.4 (Utility FUnCtion) We shall say that a proper concave function $U: \mathbb{R}^{d} \rightarrow[-\infty, \infty)$ is a (multivariate) utility function if

(i) $C_{U}:=\operatorname{cl}(\operatorname{dom}(U))$ is a convex cone which contains the non-negative orthant $\mathbb{R}_{+}^{d}$; and

(ii) $U$ is increasing with respect to the preorder induced by the closed convex cone $C_{U}$.

We call $C_{U}$ the support (or support cone) of $U$, and say that $U$ is supported on $C_{U}$.

Throughout the whole paper the agent's utility function $U$ is assumed to be supported on $\mathbb{R}_{+}^{d}$, the extended utility function $\tilde{U}$ defined by (1.1) is therefore supported on $\mathbb{R}_{+}^{D}$. It is shown in [CO10] (Proposition 3.1) that under Assumption 3.1 the value function $\bar{u}$ is a utility function which is supported on $\mathbb{R}^{D} \cap\left(-\mathcal{A}_{T}^{0}\right)$, a cone which is strictly larger than $\mathbb{R}_{+}^{D}$. It follows that $\bar{u}$ is finite on $\mathcal{I}:=\operatorname{int}\left(\mathbb{R}^{D} \cap\left(-\mathcal{A}_{T}^{0}\right)\right)$, a fact that we will use later.

We now review the analogues of the well known "Inada conditions" for the case of a multivariate utility function. For the proofs of the results, as well as for a more detailed discussion, we refer the reader to $[\mathrm{CO} 10]$.

The first condition, which we recall from [Roc72], is well known within the field of convex analysis.

Definition 2.5 A proper concave function $U: \mathbb{R}^{d} \rightarrow[-\infty, \infty)$ is said to be essentially smooth if

(i) $\operatorname{int}(\operatorname{dom}(U))$ is non-empty;

(ii) $U$ is differentiable throughout $\operatorname{int}(\operatorname{dom}(U))$;

(iii) $\lim _{i \rightarrow \infty}\left|\nabla U\left(x_{i}\right)\right|=+\infty$ whenever $x_{1}, x_{2}, \ldots$ is a sequence in $\operatorname{int}(\operatorname{dom}(U))$ converging to a boundary point of $\operatorname{int}(\operatorname{dom}(U))$.

A proper convex function $V$ is said to be essentially smooth if $-V$ is essentially smooth.

LEMMA 2.2 [CO10, Lemma 2.12] Let $U$ be a proper concave function which is essentially smooth and strictly concave on $\operatorname{int}(\operatorname{dom}(U))$. Then $U^{*}$ is strictly convex on $\operatorname{int}\left(\operatorname{dom}\left(U^{*}\right)\right)$, and essentially smooth. Moreover, the maps $\nabla U: \operatorname{int}(\operatorname{dom}(U)) \rightarrow \operatorname{int}\left(\operatorname{dom}\left(U^{*}\right)\right)$ and $\nabla U^{*}: \operatorname{int}\left(\operatorname{dom}\left(U^{*}\right)\right) \rightarrow-\operatorname{int}(\operatorname{dom}(U))$ are bijective and $(\nabla U)^{-1}=-\nabla U^{*}$.

The next condition was first introduced by [CO10] and it plays an important role in the paper.

DEFINITION 2.6 We say that a utility function $U$ is asymptotically satiable if for all $\epsilon>0$ there exists an $x \in \mathbb{R}^{d}$ such that $\partial(\operatorname{cl}(U))(x) \cap[0, \epsilon)^{d} \neq \emptyset$.

LEMma 2.3 [CO10, Lemma 2.14] A sufficient condition for asymptotic satiability of $U$ is that for all $\epsilon>0$ there exists an $x \in \operatorname{int}(\operatorname{dom}(U))$ such that $\partial U(x) \cap[0, \epsilon)^{d} \neq \emptyset$. If $U$ is closed, or essentially smooth then the condition is both necessary and sufficient for asymptotic satiability.

The next proposition clarifies the effects of asymptotic satiability on the dual function.

\footnotetext{
$\dagger$ A concave functional is weakly upper semi-continuous if and only if it is upper semi-continuous with respect to the original topology $\tau$
} 
Proposition 2.1 [CO10, Proposition 2.15] Let $U$ be a utility function. The following conditions are equivalent:

(i) $U$ is asymptotically satiable;

(ii) $0 \in \operatorname{cl}\left(\operatorname{dom}\left(U^{*}\right)\right)$;

(iii) $\operatorname{cl}\left(\operatorname{dom}\left(U^{*}\right)\right)=\left(C_{U}\right)^{*}$; and

(iv) $\operatorname{cl}\left(\operatorname{dom}\left(U^{*}\right)\right)$ is a convex cone.

If $U$ is asymptotically satiable then we define the closed convex cone $C_{U^{*}}:=\mathrm{cl}\left(\operatorname{dom}\left(U^{*}\right)\right)$, so that condition (iii) can be written more succinctly as $C_{U^{*}}=\left(C_{U}\right)^{*}$.

We note that for a utility function $U$ supported on $\mathbb{R}_{+}^{D}$, the previous proposition states that if $U$ is asymptotically satiable then $\operatorname{cl}\left(\operatorname{dom}\left(U^{*}\right)\right)=\mathbb{R}_{+}^{D}$.

Corollary 2.1 [CO10, Corollary 2.16] Let $U: \mathbb{R}^{d} \rightarrow[-\infty, \infty)$ be a utility function which is supported on $\mathbb{R}_{+}^{d}$, and which satisfies Assumption 1.1. Recall that by definition of the dual function we have

$$
U^{*}\left(x^{*}\right) \geq U(x)-\left\langle x, x^{*}\right\rangle
$$

for all $x, x^{*} \in \mathbb{R}^{d}$. If $x^{*} \in \operatorname{int}\left(\mathbb{R}_{+}^{d}\right)$ then we have equality in (2.2) if and only if $x=I\left(x^{*}\right):=-\nabla U^{*}\left(x^{*}\right)$.

Given $D \geq d$, define $\tilde{U}: \mathbb{R}^{D} \rightarrow[-\infty, \infty)$ by (1.1). Again, by definition of the dual function we have

$$
\tilde{U}^{*}\left(x^{*}\right) \geq \tilde{U}(x)-\left\langle x, x^{*}\right\rangle,
$$

for all $x, x^{*} \in \mathbb{R}^{D}$. Define $P: \mathbb{R}^{D} \rightarrow \mathbb{R}^{d}$ by

$$
P\left(x_{1}, \ldots, x_{d}, x_{d+1}, \ldots, x_{D}\right):=\left(x_{1}, \ldots, x_{d}\right),
$$

and $\tilde{I}: \operatorname{int}\left(\mathbb{R}_{+}^{d}\right) \times \mathbb{R}_{+}^{D-d} \rightarrow \operatorname{int}\left(\mathbb{R}_{+}^{d}\right) \times \mathbb{R}_{+}^{D-d}$ by

$$
\tilde{I}\left(x^{*}\right):=\left(-\nabla U^{*}\left(P\left(x^{*}\right)\right), \underline{0}\right),
$$

where $\underline{0}$ denotes the zero vector in $\mathbb{R}^{D-d}$. Then, (i) if $x^{*} \in \operatorname{int}\left(\mathbb{R}_{+}^{d}\right) \times \mathbb{R}_{+}^{D-d}$ then we have equality in (2.3) whenever $x=\tilde{I}\left(x^{*}\right)$ and (ii) if $x^{*} \in \operatorname{int}\left(\mathbb{R}_{+}^{D}\right)$ then there is equality in (2.3) if and only if $x=\tilde{I}\left(x^{*}\right)$.

\subsection{Euclidean vector measures}

A function $m$ from a field $\mathscr{F}$ of subsets of a set $\Omega$ to a Banach space $\mathscr{X}$ is called a finitely additive vector measure, or simply a vector measure if $m\left(A_{1} \cup A_{2}\right)=m\left(A_{1}\right)+m\left(A_{2}\right)$, whenever $A_{1}$ and $A_{2}$ are disjoint members of $\mathscr{F}$. In this paper, we will be concerned with the special case where $\mathscr{X}=\mathbb{R}^{D}$; we refer to the associated vector measure as a "Euclidean vector measure", or simply a "Euclidean measure". Let us recall a few definitions from the classical, one-dimensional setting. The total variation of a (finitely additive) measure $m: \mathscr{F} \rightarrow \mathbb{R}$ is the function $|m|: \mathscr{F} \rightarrow[0, \infty]$ defined by

$$
|m|(A):=\sup \sum_{j=1}^{n}\left|m\left(A_{j}\right)\right|,
$$

where the supremum is taken over all finite sequences $\left(A_{j}\right)_{j=1}^{n}$ of disjoint sets in $\mathscr{F}$ with $A_{j} \subseteq A$. A measure $m$ is said to have bounded total variation if $|m|(\Omega)<\infty$. A measure $m$ is said to be bounded if $\sup \{|m(A)|: A \in \mathscr{F}\}<\infty$. A measure $m$ is said to be purely finitely additive if $0 \leq \mu \leq|m|$ and $\mu$ is countably additive imply that $\mu=0$. A measure $m$ is said to be weakly absolutely continuous with respect to $\mathbb{P}$ if $m(A)=0$ whenever $A \in \mathscr{F}$ and $\mathbb{P}(A)=0$.

We turn now to the $D$-dimensional case. A Euclidean measure $m$ can be decomposed into its onedimensional coordinate measures $m_{i}: \mathscr{F} \rightarrow \mathbb{R}$ by defining $m_{i}(A):=\left\langle e^{i}, m(A)\right\rangle$, where $e^{i}$ is the $i$-th canonical basis vector of $\mathbb{R}^{D}$. In this way, $m(A)=\left(m_{1}(A), \ldots, m_{D}(A)\right)$ for every $A \in \mathscr{F}$. We shall say that a Euclidean measure $m$ is bounded, purely finitely additive or weakly absolutely continuous with respect to $\mathbb{P}$ if each of its coordinate measures is bounded, purely finitely additive or weakly absolutely continuous with respect to $\mathbb{P}$. 
We denote ba $\left(\mathbb{R}^{D}\right)=\mathrm{ba}\left(\Omega, \mathscr{F}_{T}, \mathbb{P} ; \mathbb{R}^{D}\right)$ the vector space of bounded Euclidean measures $m: \mathscr{F}_{T} \rightarrow$ $\mathbb{R}^{D}$ which are weakly absolutely continuous with respect to $\mathbb{P}$, and ca $\left(\mathbb{R}^{D}\right)$ the subspace of countably additive members of $\mathrm{ba}\left(\mathbb{R}^{D}\right)$. Equipped with the norm

$$
\|m\|_{\mathrm{ba}\left(\mathbb{R}^{D}\right)}:=\sum_{i=1}^{D}\left|m_{i}\right|(\Omega),
$$

the spaces ba $\left(\mathbb{R}^{D}\right)$ and ca $\left(\mathbb{R}^{D}\right)$ are Banach spaces.

Let ba $\left(\mathbb{R}_{+}^{D}\right)$ denote the convex cone of $\mathbb{R}_{+}^{D}$-valued measures within ba $\left(\mathbb{R}^{D}\right)$. The next proposition is an immediate extension of its univariate counterpart.

Proposition 2.2 Given any $m \in \mathrm{ba}\left(\mathbb{R}^{D}\right)$ there exists a unique Yosida-Hewitt decomposition $m=$ $m^{c}+m^{p}$ where $m^{c} \in \mathrm{ca}\left(\mathbb{R}^{D}\right)$ and $m^{p}$ is purely finitely additive. If $m \in \mathrm{ba}\left(\mathbb{R}_{+}^{D}\right)$ then $m^{c}, m^{p} \in \mathrm{ba}\left(\mathbb{R}_{+}^{D}\right)$.

It is well known that $L^{\infty}\left(\mathbb{R}^{D}\right)^{*}$, the set of linear functionals on the space of (essentially) bounded $\mathbb{R}^{D}$-valued random variables, can be identified with ba $\left(\mathbb{R}^{D}\right)$. Another standard result in functional analysis is that $\left(\mathrm{ba}\left(\mathbb{R}^{D}\right),\|\cdot\|_{\mathrm{ba}\left(\mathbb{R}^{D}\right)}\right)$ has a $\sigma\left(\mathrm{ba}\left(\mathbb{R}^{D}\right), L^{\infty}\left(\mathbb{R}^{D}\right)\right)$-compact unit ball. For any $m \in$ ba $\left(\mathbb{R}^{D}\right)$ we will denote

$$
m(X):=\int_{\Omega}\langle X, \mathrm{~d} m\rangle:=\sum_{i=1}^{D} \int_{\Omega} X_{i} \mathrm{~d} m_{i} .
$$

Given $x \in \mathbb{R}^{D}$ and $A \in \mathscr{F}_{T}$ we clearly have $m\left(x \chi_{A}\right)=\langle x, m(A)\rangle$. In the special case where $A=\Omega$, we have $m(x)=\langle x, m(\Omega)\rangle$.

Let $L^{0}\left(\mathbb{R}_{+}^{D}\right)$ and $L^{\infty}\left(\mathbb{R}_{+}^{D}\right)$ denote respectively the convex cones of random variables in $L^{0}\left(\mathbb{R}^{D}\right)$ and $L^{\infty}\left(\mathbb{R}^{D}\right)$ which are $\mathbb{R}_{+}^{D}$-valued a.s. Note that if $m \in \mathrm{ba}\left(\mathbb{R}_{+}^{D}\right)$ and $X \in L^{\infty}\left(\mathbb{R}_{+}^{D}\right)$ then $m(X) \geq 0$ (see [RR83, Theorem 4.4.13]). This observation allows us to extend the definition of $m(X)$ to cover the case where $m \in \mathrm{ba}\left(\mathbb{R}_{+}^{D}\right)$ and $X \in L^{0}\left(\mathbb{R}_{+}^{D}\right)$ (not necessarily bounded from above) by setting

$$
m(X):=\sup _{n \in \mathbb{N}} m(X \wedge(n \mathbf{1}))
$$

where $\left(x_{1}, \ldots, x_{D}\right) \wedge\left(y_{1}, \ldots, y_{D}\right):=\left(x_{1} \wedge y_{1}, \ldots, x_{D} \wedge y_{D}\right)$. It is trivial that $(2.6)$ is consistent with the definition of $m(X)$ for $X \in L^{\infty}\left(\mathbb{R}^{D}\right)$. Furthermore, the supremum in (2.6) can be replaced by a limit, since the sequence of numbers is increasing. It follows that given $m_{1}, m_{2} \in \mathrm{ba}\left(\mathbb{R}_{+}^{D}\right), \lambda_{1}, \lambda_{2}, \mu_{1}, \mu_{2} \geq 0$ and $X_{1}, X_{2} \in L^{0}\left(\mathbb{R}_{+}^{D}\right)$, we have

$$
\left(\lambda_{1} m_{1}+\lambda_{2} m_{2}\right)\left(\mu_{1} X_{1}+\mu_{2} X_{2}\right)=\lambda_{1} \mu_{1} m_{1}\left(X_{1}\right)+\lambda_{1} \mu_{2} m_{1}\left(X_{2}\right)+\lambda_{2} \mu_{1} m_{2}\left(X_{1}\right)+\lambda_{2} \mu_{2} m_{2}\left(X_{2}\right) .
$$

Given $m \in \operatorname{ca}\left(\mathbb{R}^{D}\right)$ and $X \in L^{\infty}\left(\mathbb{R}^{D}\right)$ we have $m(X)=\mathrm{E}\left[\left\langle X, \frac{\mathrm{d} m}{\mathrm{~d} \mathbb{P}}\right\rangle\right]$, where $\frac{\mathrm{d} m}{\mathrm{~d} \mathbb{P}}$ is the vector of Radon-Nikodym derivatives. It is easy to show that this property is also true under the extended definition (2.6).

More details on finitely additive measures (which are sometimes referred to as charges) can be found in [RR83].

\section{Utility maximization problem with random endowment}

In this section we will elaborate on the main optimization problem that was defined in (3.2), with a particular focus on the issue of existence of a solution. We start by investigating some useful properties of the value function in Proposition 3.1. We then proceed by dualizing the problem in Section 3.1, using some convex duality techniques that are commonly used in optimization (see, for example, [Bou02], [OZ09], [CO10] among others). Lemma 3.1 will give another convenient representation of the dual functional, while Theorem 3.1 will establish the absence of duality gap and the existence of a solution to the dual problem under some rather weak conditions on $\mathcal{E}$ (see condition (3.1) below). Finally, in Section 3.2 , we show the existence of a solution to the primal problem in Theorem 3.2 under the additional assumptions of asymptotic satiability of the value function and boundedness of the endowment.

For technical reasons that will be clear later in the proofs, we will mainly consider endowments of this form: $\mathcal{E} \in L^{0}\left(\mathbb{R}^{D}, \mathcal{F}_{T}\right)$ and there exist $x^{\prime}, x^{\prime \prime} \in \mathcal{I}:=\operatorname{int}\left(-\mathcal{A}_{T}^{0} \cap \mathbb{R}^{D}\right)$ and $X^{\prime \prime} \in \mathcal{A}_{T}^{0}$ such that

$$
x^{\prime} \preceq \mathcal{E} \preceq x^{\prime \prime}+X^{\prime \prime}
$$


We call $\mathcal{O}$ the convex set of endowments satisfying (3.1) for some $x^{\prime}, x^{\prime \prime} \in \mathcal{I}$ and $X^{\prime \prime}$.

For any $\mathcal{E} \in \mathcal{O}$ we define the primal optimization problem as

$$
u(\mathcal{E}):=\sup \left\{\mathbb{E}[\tilde{U}(X+\mathcal{E})]: X \in \mathcal{A}_{T}^{0}\right\}
$$

When $\mathcal{E}=x$ is deterministic, this reduces to the formulation in [CO10]:

$$
\bar{u}(x):=\sup \left\{\mathbb{E}[\tilde{U}(X)]: X \in \mathcal{A}_{T}^{x}\right\} .
$$

We denote $\operatorname{dom}(u):=\left\{\mathcal{E} \in L^{0}\left(\mathbb{R}^{D}\right): u(\mathcal{E})>-\infty\right\}$ and $\tilde{\mathcal{A}}_{T}^{0}:=\left\{X \in \mathcal{A}_{T}^{0}: \exists \epsilon>0: X+\epsilon \mathbf{1} \in \mathcal{A}_{T}^{0}\right\}$. The set $\tilde{\mathcal{A}}_{T}^{0}$ is clearly not empty as it contains the constants in the (strictly) negative orthant. The following mild assumption is fairly natural in any optimization problem (compare [CO10, Assumption 1.2]).

Assumption $3.1 \bar{u}(x)<+\infty$ for some $x \in \operatorname{int}(\operatorname{dom}(\bar{u}))$.

Under this assumption, we can rephrase condition (3.1) as follows: $x^{\prime} \preceq \mathcal{E} \preceq x^{\prime \prime}+X^{\prime \prime}$ for some initial portfolios $x^{\prime}, x^{\prime \prime}$ in $\operatorname{int}(\operatorname{dom} \bar{u})$ and some final portfolio $X^{\prime \prime} \in \mathcal{A}_{T}^{0}$. Indeed, it has been established in $\left[\right.$ CO10, Proposition 3.1] that, under Assumption 3.1, one has $\operatorname{cl}(\operatorname{dom} \bar{u})=-\mathcal{A}_{T}^{0} \cap \mathbb{R}^{D}$.

REMARK 3.1 Take any $\mathcal{E} \in \mathcal{O}$. Notice that $u(\mathcal{E}-\epsilon \mathbf{1}) \geq \mathbb{E}[\tilde{U}(X+\mathcal{E}-\epsilon \mathbf{1})] \geq \mathbb{E}\left[\tilde{U}\left(X+x^{\prime}-\epsilon \mathbf{1}\right)\right]$ for all $X \in \mathcal{A}_{T}^{0}$, so that $u(\mathcal{E}-\epsilon \mathbf{1}) \geq \bar{u}\left(x^{\prime}-\epsilon \mathbf{1}\right)>-\infty$ for some $\epsilon>0$ since $x^{\prime} \in \mathcal{I}$. This simple observation will be used in the proof of the following proposition.

Proposition 3.1 The value function $u: L^{0}\left(\mathbb{R}^{D}\right) \rightarrow[-\infty, \infty]$ has the following properties:

(i) $u$ is concave on $\mathcal{O}$ and increasing with respect to $L^{0}\left(\mathbb{R}_{+}^{D}\right)$.

(ii) $u(\mathcal{E}) \in \mathbb{R}$ for any $\mathcal{E} \in \mathcal{O}$, so that in particular $\mathcal{O} \subseteq \operatorname{dom}(u)$;

(iii) $u(\mathcal{E})<\infty$ for any $\mathcal{E} \in \mathcal{O} \cap L^{\infty}\left(\mathbb{R}^{D}\right)$;

(iv) $\operatorname{cl}(\operatorname{dom}(u))=-\operatorname{cl}\left(\mathcal{A}_{T}^{0}\right)$ in the topology of convergence in probability;

(v) $u$ is increasing w.r.t. the preorder generated by $\operatorname{dom}(u)$. If $U$ is l.s.c. then $u$ is also increasing w.r.t. the preorder generated by $\mathrm{cl}(\operatorname{dom}(u))$.

Proof. (i) Concavity follows from the fact that $\mathcal{A}_{T}^{0}$ is convex and $\tilde{U}$ is concave. The second property follows from the same property for $U$.

(ii) Observe that $u(\mathcal{E}) \geq \mathbb{E}[\tilde{U}(X+\mathcal{E})] \geq \mathbb{E}\left[\tilde{U}\left(X+x^{\prime}\right)\right]$ for all $X \in \mathcal{A}_{T}^{0}$, so that $u(\mathcal{E}) \geq u\left(x^{\prime}\right)>-\infty$ since $x^{\prime} \in \mathcal{I} \subseteq \operatorname{int}(\operatorname{dom}(\bar{u}))$, where we recall that $\bar{u}$ is the restriction of the value function $u$ on $\mathbb{R}^{D}$. Also note that $u(\mathcal{E}) \leq \mathbb{E}\left[\tilde{U}\left(X+x^{\prime}+X^{\prime \prime}\right)\right] \leq \bar{u}\left(x^{\prime \prime}\right)<\infty$ whenever $x^{\prime \prime} \in \mathcal{I}$ (See Section 2.2). Hence $u(\mathcal{E}) \in \mathbb{R}$.

(iii) We show that $u(\mathcal{E})<\infty$ for any $\mathcal{E} \in \mathcal{O} \cap L^{\infty}$. Suppose for a contradiction that there exists some $\tilde{\mathcal{E}} \in L^{\infty}$ such that $u(\tilde{\mathcal{E}})=\infty$. Let $\mathcal{E} \in \mathcal{O}$, so that $u(\mathcal{E})<\infty$. We can find an $a>0$ such that $\mathcal{E}_{1}:=\mathcal{E}+a \mathbf{1} \succeq \tilde{\mathcal{E}}$ a.s.. We have $u\left(\mathcal{E}_{1}\right) \geq u(\tilde{\mathcal{E}})=\infty$.

By Remark 3.1 there exists an $\epsilon>0$ such that $\mathcal{E}_{0}:=\mathcal{E}-\epsilon \mathbf{1} \in \operatorname{dom}(u)$, so that $u\left(\mathcal{E}_{0}\right)>-\infty$. We also have $u\left(\mathcal{E}_{0}\right) \leq u(\mathcal{E})<\infty$, hence $u\left(\mathcal{E}_{0}\right) \in \mathbb{R}$. This implies that we may find an $X_{0} \in \mathcal{A}_{T}^{0}$ such that $\mathbb{E}\left[\tilde{U}\left(X_{0}+\mathcal{E}_{0}\right)\right]=: c \in \mathbb{R}$. Since $u\left(\mathcal{E}_{1}\right)=\infty$, given any $R \in \mathbb{R}$ we may also find an $X_{1} \in \mathcal{A}_{T}^{0}$ such that $\mathbb{E}\left[\tilde{U}\left(X_{1}+\mathcal{E}_{1}\right)\right] \geq R$. Define $\lambda:=\epsilon /(a+\epsilon)$ and $X:=(1-\lambda) X_{0}+\lambda X_{1}$. So we have

$$
\begin{aligned}
u(\mathcal{E}) & \geq \mathbb{E}[\tilde{U}(X+\mathcal{E})]=\mathbb{E}\left[\tilde{U}\left((1-\lambda)\left(X_{0}+\mathcal{E}_{0}\right)+\lambda\left(X_{1}+\mathcal{E}_{1}\right)\right)\right] \\
& \geq(1-\lambda) \mathbb{E}\left[\tilde{U}\left(X_{0}+\mathcal{E}_{0}\right)\right]+\lambda \mathbb{E}\left[\tilde{U}\left(X_{1}+\mathcal{E}_{1}\right)\right] \geq(1-\lambda) c+\lambda R
\end{aligned}
$$

which is a contradiction since $R$ can be taken arbitrarily large.

(iv) Take $X_{0} \in \tilde{\mathcal{A}}_{T}^{0}$. There exists $\epsilon>0$ such that $X_{0}+\epsilon \mathbf{1} \in \mathcal{A}_{T}^{0}$, then

$$
u\left(-X_{0}\right) \geq \mathbb{E}[\tilde{U}(\epsilon \mathbf{1})]>-\infty
$$


hence $-X_{0} \in \operatorname{dom}(u)$, so $-\tilde{\mathcal{A}}_{T}^{0} \subseteq \operatorname{dom}(u)$.

Suppose that $\mathcal{E} \in \operatorname{dom}(u)$. Necessarily then $\mathcal{A}_{T}^{\mathcal{E}} \cap L^{0}\left(\mathbb{R}_{+}^{D}\right) \neq \emptyset$, where $\mathcal{A}_{T}^{\mathcal{E}}:=\mathcal{E}+\mathcal{A}_{T}^{0}=\left\{Y \in L^{0}: Y=\right.$ $\left.X+\mathcal{E}, X \in \mathcal{A}_{T}^{0}\right\}$. Take $X \in \mathcal{A}_{T}^{\mathcal{E}} \cap L^{0}\left(\mathbb{R}_{+}^{D}\right)$, then $0=X-X \in \mathcal{A}_{T}^{\mathcal{E}}-L^{0}\left(\mathbb{R}_{+}^{D}\right) \subseteq \mathcal{A}_{T}^{\mathcal{E}}$, hence $0 \in \mathcal{A}_{T}^{\mathcal{E}}$, which implies $\mathcal{E} \in-\mathcal{A}_{T}^{0}$. So

$$
-\tilde{\mathcal{A}}_{T}^{0} \subseteq \operatorname{dom}(u) \subseteq-\mathcal{A}_{T}^{0}
$$

and the claim follows from $\operatorname{cl}\left(-\tilde{\mathcal{A}}_{T}^{0}\right)=\operatorname{cl}\left(-\mathcal{A}_{T}^{0}\right)$. To see the last equality, remark first that $\operatorname{cl}\left(\tilde{\mathcal{A}}_{T}^{0}\right) \subseteq$ $\operatorname{cl}\left(\mathcal{A}_{T}^{0}\right)$. Now take $X \in \operatorname{cl}\left(\mathcal{A}_{T}^{0}\right)$, then (up to a subsequence) there exists $\left(X_{n}\right)_{n \geq 0} \in \mathcal{A}_{T}^{0}$ such that $X_{n} \rightarrow X$ almost surely. Let $\left(\epsilon_{n}\right)_{n \geq 0}>0$ be such that $\epsilon_{n} \rightarrow 0$ and remark that $Y_{n}:=X_{n}-\epsilon_{n} \mathbf{1}$ belongs to $\tilde{\mathcal{A}}_{T}^{0}$ and $Y_{n} \rightarrow X$ almost surely. Hence $Y_{n} \rightarrow X$ in probability yielding $X \in \operatorname{cl}\left(\tilde{\mathcal{A}}_{T}^{0}\right)$.

(v) We only prove the second part of the claim. Take $\mathcal{E} \in L^{0}\left(\mathbb{R}^{D}\right)$ such that $u(\mathcal{E})<\infty$ and $\mathcal{E}_{1} \in \operatorname{cl}(\operatorname{dom}(u))$, so by property (iv) there exists $\left(Y_{n}\right)_{n \geq 0} \in \mathcal{A}_{T}^{0}$ such that $Y_{n} \rightarrow-\mathcal{E}_{1}$ almost surely (up to a subsequence). By definition, for any $\epsilon>0$ there exists a $X \in \mathcal{A}_{T}^{0}$ such that $\mathbb{E}[\tilde{U}(X+\mathcal{E})] \geq u(\mathcal{E})-\epsilon$. Since $X+Y_{n} \in \mathcal{A}_{T}^{0}$ we have that $u\left(\mathcal{E}+\mathcal{E}_{1}\right) \geq \mathbb{E}\left[\tilde{U}\left(X+Y_{n}+\mathcal{E}+\mathcal{E}_{1}\right)\right]$ for all $n \in \mathbb{N}$, hence

$$
\begin{aligned}
u\left(\mathcal{E}+\mathcal{E}_{1}\right) & \geq \liminf _{n} \mathbb{E}\left[\tilde{U}\left(X+Y_{n}+\mathcal{E}+\mathcal{E}_{1}\right)\right] \geq \mathbb{E}\left[\liminf _{n} \tilde{U}\left(X+Y_{n}+\mathcal{E}+\mathcal{E}_{1}\right)\right] \\
& \geq \mathbb{E}[\tilde{U}(X+\mathcal{E})] \geq u(\mathcal{E})-\epsilon
\end{aligned}
$$

where we used the fact that $\tilde{U}$ is l.s.c. Since $\epsilon$ is arbitrary the claim follows.

If $u(\mathcal{E})=\infty$, then we can find an $X \in \mathcal{A}_{T}^{0}$ such that $\mathbb{E}[\tilde{U}(X+\mathcal{E})] \geq R$ for any $R>0$. With the same arguments as above we can say that $u\left(\mathcal{E}+\mathcal{E}_{1}\right) \geq R$, hence $u\left(\mathcal{E}+\mathcal{E}_{1}\right)=u(\mathcal{E})=\infty$.

\subsection{Dual representation of the optimization problem}

In this section we show that the value function of our optimization problem with random endowment can be represented as the value function of a suitably defined dual minimization problem. To do so, let us define the functional

$$
\mathbb{U}_{\mathcal{E}}(X):=\mathbb{E}[\tilde{U}(X+\mathcal{E})]
$$

and its dual

$$
\mathbb{U}_{\mathcal{E}}^{*}(m):=\sup _{X \in L^{\infty}\left(\mathbb{R}^{D}\right)}\left[\mathbb{U}_{\mathcal{E}}(X)-m(X)\right]
$$

Lemma 3.1 If $\mathcal{E} \in \mathcal{O}$ then we have the following representation:

$$
\mathbb{U}_{\mathcal{E}}^{*}(m)=\left\{\begin{aligned}
\mathbb{E}\left[\tilde{U}^{*}\left(\frac{d m^{c}}{d P}\right)\right]+m(\mathcal{E}) & \text { if } m \in \mathrm{ba}\left(\mathbb{R}_{+}^{D}\right) \\
\infty & \text { otherwise }
\end{aligned}\right.
$$

Proof. Remark first that since $\mathbb{U}_{0}$ is increasing with respect to the preorder induced by $L^{\infty}\left(\mathbb{R}_{+}^{D}\right)$ it follows from Lemma 2.1 that $\operatorname{dom}\left(\mathbb{U}_{0}^{*}\right) \subseteq L^{\infty}\left(\mathbb{R}_{+}^{D}\right)^{*}=\mathrm{ba}\left(\mathbb{R}_{+}^{D}\right)$.

Now take $m \in \mathrm{ba}\left(\mathbb{R}_{+}^{D}\right)$ and define

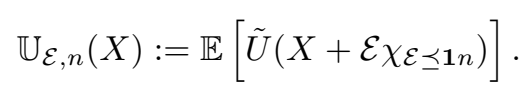

It is clear that by monotone convergence one has

$$
\lim _{n \rightarrow \infty} \mathbb{U}_{\mathcal{E}, n}(X)=\sup _{n} \mathbb{U}_{\mathcal{E}, n}(X)=\mathbb{U}_{\mathcal{E}}(X)
$$


Thus we get

$$
\begin{aligned}
\mathbb{U}_{\mathcal{E}}^{*}(m) & =\sup _{X \in L^{\infty}\left(\mathbb{R}^{D}\right)}\left[\mathbb{U}_{\mathcal{E}}(X)-m(X)\right] \\
& =\sup _{X \in L^{\infty}\left(\mathbb{R}^{D}\right)} \sup _{n}\left[\mathbb{U}_{\mathcal{E}, n}(X)-m(X)\right] \\
& =\sup _{n} \sup _{X \in L^{\infty}\left(\mathbb{R}^{D}\right)}\left[\mathbb{U}_{\mathcal{E}, n}(X)-m(X)\right] \\
& =\lim _{n \rightarrow \infty} \sup _{X \in L^{\infty}\left(\mathbb{R}^{D}\right)}\left[\mathbb{U}_{0}(X+\mathcal{E} \chi(\mathcal{E} \preceq n \mathbf{1}))-m(X+\mathcal{E} \chi(\mathcal{E} \preceq n \mathbf{1}))\right]+m\left(\mathcal{E} \chi_{\mathcal{E} \preceq \mathbf{1} n}\right) \\
& =\lim _{n \rightarrow \infty} \sup _{\tilde{X} \in L^{\infty}\left(\mathbb{R}^{D}\right)}\left[\mathbb{U}_{0}(\tilde{X})-m(\tilde{X})\right]+m\left(\mathcal{E} \chi_{\mathcal{E} \preceq \mathbf{1} n}\right) \\
& =\mathbb{U}_{0}^{*}(m)+\lim _{n \rightarrow \infty} m(\mathcal{E} \chi \mathcal{E} \preceq 1 n) \\
& =\mathbb{U}_{0}^{*}(m)+m(\mathcal{E}) .
\end{aligned}
$$

Now that we have isolated the contribution of the random endowment, it suffices to study the case of zero endowment to conclude the proof. This has already been done in [CO10], where it is shown that $\mathbb{U}_{0}^{*}(m)=\mathbb{U}_{0}^{*}\left(m^{c}\right)=\mathbb{E}\left[\tilde{U}^{*}\left(\frac{d m^{c}}{d P}\right)\right]$, which yields the claim.

REMARK 3.2 A consequence of previous Lemma 3.1 is that if $\mathcal{E} \in \mathcal{O}$ then $\operatorname{dom}\left(\mathbb{U}_{\mathcal{E}}^{*}\right)=\operatorname{dom}\left(\mathbb{U}_{0}^{*}\right)$. Measures in this set are sometimes said to have finite relative entropy (see, for example, [OZ09]).

Consider now the abstract maximization problem

$$
\sup _{X \in \mathcal{C}} \mathbb{U}_{\mathcal{E}}(X)
$$

where

$$
\mathcal{C}:=L^{\infty}\left(\mathbb{R}^{D}\right) \cap \mathcal{A}_{T}^{0}
$$

It is immediately clear that

$$
\sup _{X \in \mathcal{C}} \mathbb{U}_{\mathcal{E}}(X) \leq u(\mathcal{E})
$$

Its abstract dual problem is defined as

$$
\inf _{m \in \mathcal{D}} \mathbb{U}_{\mathcal{E}}^{*}(m)
$$

where $\mathbb{U}_{\mathcal{E}}^{*}$ is defined as in $(3.3)$ and

$$
\mathcal{D}:=\left\{m \in \operatorname{ba}\left(\mathbb{R}^{D}\right): m(X) \leq 0 \text { for all } X \in \mathcal{C}\right\} .
$$

Since $-L^{\infty}\left(\mathbb{R}_{+}^{D}\right) \subseteq \mathcal{C}$, one clearly has $\mathcal{D} \subseteq$ ba $\left(\mathbb{R}_{+}^{D}\right)$. We introduce the Lagrangian $L(X, m):=\mathbb{U}_{\mathcal{E}}(X)-$ $m(X)$ and note that

$$
\sup _{X \in \mathcal{C}} \mathbb{U}_{\mathcal{E}}(X) \leq \sup _{X \in L^{\infty}} \inf _{m \in \mathcal{D}} L(X, m) \leq \inf _{m \in \mathcal{D}} \sup _{X \in L^{\infty}} L(X, m)=\inf _{m \in \mathcal{D}} \mathbb{U}_{\mathcal{E}}^{*}(m)
$$

REMARK 3.3 It is important to notice that, given any $Z \in \mathcal{Z}^{s}$ we can construct a corresponding $m \in \mathrm{ca}\left(\mathbb{R}_{+}^{D}\right)$ by setting $m(A):=\mathbb{E}\left[Z_{T} \mathbf{1}_{A}\right]$ for each $A \in \mathcal{F}_{T}$. We call $m^{Z}$ the measure associated to the price process $Z$. We have that

$$
\mathcal{Z}^{s} \subseteq \mathcal{D} \cap \operatorname{ca}\left(\mathbb{R}_{+}^{D}\right) \subseteq \mathcal{Z}
$$

To see that, begin with the first inclusion (that was already established in [CO10, Remark 3.10]) : Take $Z \in \mathcal{Z}^{s}$ and $X \in \mathcal{C}$. Then $\mathbb{E}\left[\left\langle X, Z_{T}\right\rangle\right]=m^{Z}(X) \leq 0$ by Theorem 2.1, where $m^{Z} \in \operatorname{ca}\left(\mathbb{R}_{+}^{D}\right)$.

For the second inclusion, take $m \in \mathcal{D} \cap \mathrm{ca}\left(\mathbb{R}_{+}^{D}\right)$, so that $m(X) \leq 0$ for any $X \in \mathcal{C}$. Take any $X \in$ $L^{\infty}\left(-K_{t}, \mathcal{F}_{t}\right)$ for some $t$, then $X \in \mathcal{A}_{T}^{0}$ (consider the strategy that just trades at time $t$ for an amount equal to $X)$. So $X \in \mathcal{C}$ and then $m(X) \leq 0$, which implies $Z_{t}^{m}:=\mathbb{E}\left[\frac{d m}{d P} \mid \mathcal{F}_{t}\right] \in K_{t}^{*}$ a.s. and so $Z^{m} \in \mathcal{Z}$. By monotone convergence, this is also true for unbounded $X$.

Define

$$
\mathcal{P}:=\left\{m \in \mathrm{ba}\left(\mathbb{R}_{+}^{D}\right): P\left(\frac{d m^{c}}{d P}\right) \text { is } \operatorname{int}\left(\mathbb{R}_{+}^{d}\right)-\text { valued a.s. }\right\}
$$

where $P$ is defined in Corollary 2.1 . 
Lemma 3.2 Suppose that $\widehat{m}$ is a minimizer for the problem $\inf _{m \in \mathcal{D}} \mathbb{U}_{\mathcal{E}}^{*}(m)$. Then $\widehat{m} \in \mathcal{P}$. If the utility function $U$ is strictly concave then the minimizer is unique.

Proof. We will use the same arguments as in [CO10], Proposition 3.9, with minor modifications. However, we will give the details of the proof for reader's convenience.

By Lemma 3.1, $\widehat{m} \in \mathrm{ba}\left(\mathbb{R}_{+}^{D}\right)$. Suppose that $\widehat{m} \notin \mathcal{P}$. By definition some of the components of $\frac{d \widehat{m}^{c}}{d P}$ are zero on a set $A \in \mathcal{F}$ with positive probability. Take $Z \in \mathcal{Z}^{s}$ and let $m^{Z}$ be its associated measure as in Remark 3.3. For $\lambda>0$ let $m_{\lambda}:=\lambda m^{Z}+\widehat{m} \in \mathcal{D}$ and $\nu_{\lambda}:=\tilde{U}^{*}\left(\frac{d m_{\lambda}^{c}}{d P}\right)$. By Lemma 2.1, $\mathbb{U}_{0}^{*}$ is decreasing with respect to the preorder induced by ba $\left(\mathbb{R}_{+}^{D}\right)$, implying that $m_{\lambda} \in \operatorname{dom}\left(\mathbb{U}_{0}^{*}\right)$. Since $\nu_{\lambda}$ is convex as a function of $\lambda$, the integrable random variables $\left(\nu_{\lambda}-\nu_{0}\right) / \lambda$ are monotone increasing in $\lambda$. By the monotone convergence theorem,

$$
\begin{aligned}
& \lim _{\lambda \rightarrow 0} \mathbb{E}\left[\chi_{A}\left(\frac{\nu_{\lambda}-\nu_{0}}{\lambda}\right)\right]=\mathbb{E}\left[\chi_{A} \lim _{\lambda \rightarrow 0}\left(\frac{\nu_{\lambda}-\nu_{0}}{\lambda}\right)\right] \\
& =\mathbb{E}\left[\chi_{A} \lim _{\lambda \rightarrow 0}\left(\frac{\tilde{U}^{*}\left(\lambda \frac{d m^{Z}}{d P}+\frac{d \hat{m}^{c}}{d P}\right)-\tilde{U}^{*}\left(\frac{d \tilde{m}^{c}}{d P}\right)}{\lambda}\right)\right] \\
& =\mathbb{E}\left[\chi_{A} \lim _{\lambda \rightarrow 0}\left(\frac{U^{*}\left(\lambda P\left(\frac{d m^{Z}}{d P}\right)+P\left(\frac{d \widehat{m}^{c}}{d P}\right)\right)-U^{*}\left(P\left(\frac{d \tilde{m}^{c}}{d P}\right)\right)}{\lambda}\right)\right]=-\infty
\end{aligned}
$$

since, being $U^{*}$ essentially smooth (by Lemma 2.2), its gradient diverges on the boundary points of its domain. Hence $\lim _{\lambda \rightarrow 0} \frac{1}{\lambda} \mathbb{E}\left[\nu_{\lambda}-\nu_{0}\right]=-\infty$. By Lemma 3.1, the optimality of $\widehat{m}$, the assumptions on the endowment and Theorem 2.1 we have that $\mathbb{E}\left[\nu_{\lambda}-\nu_{0}\right]=\mathbb{U}_{\mathcal{E}}^{*}\left(m_{\lambda}\right)-m_{\lambda}(\mathcal{E})-\mathbb{U}_{\mathcal{E}}^{*}(\widehat{m})+$ $\widehat{m}(\mathcal{E}) \geq-\lambda m^{Z}(\mathcal{E}) \geq-\lambda m^{Z}\left(x^{\prime \prime}+X^{\prime \prime}\right) \geq-\lambda m^{Z}\left(x^{\prime \prime}\right)=-\lambda\left\langle x^{\prime \prime}, \mathbb{E}\left[Z_{T}\right]\right\rangle=-\lambda\left\langle x^{\prime \prime}, Z_{0}\right\rangle>-\infty$, therefore $\frac{1}{\lambda} \mathbb{E}\left[\nu_{\lambda}-\nu_{0}\right] \geq\left\langle x^{\prime \prime}, Z_{0}\right\rangle>-\infty$ and so the limit as $\lambda \rightarrow 0$ cannot be $-\infty$, which is a contradiction.

Uniqueness follows easily from strict convexity of the dual function.

Motivated by Lemma 3.1, we define

$$
v(\mathcal{E}):=\inf _{m \in \mathcal{D}}\left\{\mathbb{E}\left[\tilde{U}^{*}\left(\frac{d m^{c}}{d P}\right)\right]+m(\mathcal{E})\right\}
$$

Take $X \in \mathcal{C}$ and $m \in \mathcal{D}$. We can consider in what follows that $X+\mathcal{E} \in L^{0}\left(\mathbb{R}_{+}^{D}\right)$ otherwise the results are trivial. We have

$$
\begin{aligned}
m(X+\mathcal{E}) & =\sup _{n} m((X+\mathcal{E}) \wedge \mathbf{1} n) \leq \sup _{n} m(X \wedge \mathbf{1} n)+\sup _{n} m(\mathcal{E} \wedge \mathbf{1} n) \\
& =m(X)+m(\mathcal{E}) \leq m(\mathcal{E})
\end{aligned}
$$

We also remark that

$$
m(X+\mathcal{E}) \geq m^{c}(X+\mathcal{E})=\mathbb{E}\left[\left\langle X+\mathcal{E}, \frac{d m^{c}}{d P}\right\rangle\right] .
$$

By combining these considerations and using the definition of the dual function we get

$$
\begin{aligned}
\mathbb{E}[\tilde{U}(X+\mathcal{E})] & \leq \mathbb{E}\left[\tilde{U}^{*}\left(\frac{d m^{c}}{d P}\right)+\left\langle\frac{d m^{c}}{d P}, X+\mathcal{E}\right\rangle\right] \\
& \leq \mathbb{E}\left[\tilde{U}^{*}\left(\frac{d m^{c}}{d P}\right)\right]+m(\mathcal{E}) .
\end{aligned}
$$

After all these preliminaries, we can finally prove the existence result.

THEOREM 3.1 If $\mathcal{E} \in \mathcal{O}$ then

$$
\sup _{X \in \mathcal{C}} \mathbb{U}_{\mathcal{E}}(X)=u(\mathcal{E})=v(\mathcal{E})=\min _{m \in \mathcal{D}} \mathbb{U}_{\mathcal{E}}^{*}(m)<\infty
$$

If the utility function is strictly concave, then the minimizer is unique. 
Proof. The proof can be split into two parts.

1. We first use the Lagrange duality theorem as reported in the Appendix of [CO10] to show that $\sup _{X \in \mathcal{C}} \mathbb{U}_{\mathcal{E}}(X)=v(\mathcal{E})=\min _{m \in \mathcal{D}} \mathbb{U}_{\mathcal{E}}^{*}(m)<\infty$. Take $\mathcal{E} \in \mathcal{O}$, let $\mathcal{X}=L^{\infty}\left(\mathbb{R}^{D}\right)$ and define the concave functional $\mathfrak{U}: \mathcal{X} \rightarrow[-\infty, \infty)$ by $\mathfrak{U}=\mathbb{U}_{\mathcal{E}}$. By Remark 3.1 there exists $\epsilon>0$ such that $-Y:=\mathcal{E}-3 \epsilon \mathbf{1} \in \operatorname{dom}(u)=-\operatorname{cl}\left(\mathcal{A}_{T}^{0}\right)$. Suppose first that $Y \in \mathcal{A}_{T}^{0}$, so by using [KS09, Lemma 3.6.7] we can find a sequence $Y_{n} \in \mathcal{C} \subseteq-\operatorname{dom}(u)$ such that $Y_{n} \rightarrow Y$.

By definition $p:=-\mathbf{1} \epsilon$ belongs to the interior of $\mathcal{C}$ (with the norm of $L^{\infty}$ ), and we can assume that $Y_{n} \succeq 2 \epsilon \mathbf{1}-\mathcal{E}$ for $n$ sufficiently large. Hence $\mathfrak{U}\left(p+Y_{n}\right)=\mathfrak{U}\left(-\mathbf{1} \epsilon+Y_{n}\right) \geq \mathbb{E}[\tilde{U}(\mathbf{1} \epsilon)]>-\infty$ if $n$ is sufficently large. By property (ii) in Proposition 3.1 we have

$$
\sup _{X \in \mathcal{C}} \mathfrak{U}(X) \leq u(\mathcal{E})<\infty
$$

This verifies the hypotheses of part 1 of Theorem A.1 in [CO10], hence the claim follows.

If we have instead $Y \in \operatorname{cl}\left(\mathcal{A}_{T}^{0}\right)$ then there exists $\tilde{Y}^{k} \in \mathcal{A}_{T}^{0}$ such that $\tilde{Y}^{k} \rightarrow Y$ a.s. (up to a subsequence) and for each $\tilde{Y}^{k}$ we can find a sequence $\tilde{Y}_{n}^{k} \in \mathcal{C}$ such that $\tilde{Y}_{n}^{k} \rightarrow \tilde{Y}^{k}$. Then by the same arguments as above we have $\mathfrak{U}\left(p+Y_{n}^{k}\right) \geq \mathbb{E}[\tilde{U}(\mathbf{1} \epsilon)]>-\infty$ for $n$ and $k$ sufficently large.

2. It remains to show that

$$
\sup _{X \in \mathcal{C}} \mathbb{U}_{\mathcal{E}}(X)=u(\mathcal{E})
$$

Clearly $\sup _{X \in \mathcal{C}} \mathbb{U}_{\mathcal{E}}(X) \leq u(\mathcal{E})$. To show the other inequality, take a sequence $X^{n} \in \mathcal{A}_{T}^{0}$ such that $\mathbb{U}_{\mathcal{E}}\left(X_{n}\right) \rightarrow u(\mathcal{E})$. By step 1 , there exists $Y \in \mathcal{C}$ such that $Y+\mathcal{E} \succeq \mathbf{1} \epsilon$, so we can assume w.l.o.g. that $X^{n}+\mathcal{E} \in \operatorname{int}\left(\mathbb{R}_{+}^{D}\right)$ for all $n$. For any $\epsilon>0$ we can find $n_{0}$ such that $\mathbb{U}_{\mathcal{E}}\left(X_{n}\right) \geq u(\mathcal{E})-\epsilon$ for all $n \geq n_{0}$. By [KS09, Lemma 3.6.7], the set $\mathcal{C}=\mathcal{A}_{T}^{0} \cap L^{\infty}$ is Fatou-dense in $\mathcal{A}_{T}^{0}{ }^{\ddagger}{ }^{\ddagger}$ Thus, for any $X^{n} \in \mathcal{A}_{T}^{0}$ there is a sequence $X_{n}^{k} \in \mathcal{C}$ such that $X_{n}^{k} \rightarrow X^{n}$, and since $U$ is continuous on $\operatorname{int}\left(\mathbb{R}_{+}^{D}\right)$ by [Roc72] Theorem 10.1, we can find $k_{0}$ such that $\mathbb{U}_{\mathcal{E}}\left(X_{n}^{k}\right) \geq \mathbb{U}_{\mathcal{E}}\left(X_{n}\right)-\epsilon$ for any $k \geq k_{0}$. This implies that $\mathbb{U}_{\mathcal{E}}\left(X_{n}^{k}\right) \geq u(\mathcal{E})-2 \epsilon$ when $n$ and $k$ are sufficiently large. Since $\epsilon$ is arbitrary we finally get the opposite inequality by letting $n$ and $k$ tend to infinity.

Uniqueness follows easily by strict concavity of the utility function.

Take $\mathcal{E} \in \mathcal{O}$ and let $\widehat{m}$ be the corresponding minimizer in the abstract dual problem above, so that

$$
v(\mathcal{E})=\mathbb{E}\left[\tilde{U}^{*}\left(\frac{d \widehat{m}^{c}}{d P}\right)\right]+\widehat{m}(\mathcal{E}) \in \mathbb{R} .
$$

For $x \in \mathbb{R}_{+}^{D}$, define

$$
\mathcal{D}(x):=\{m \in \mathcal{D}, m(\Omega)=x\} .
$$

Take $x \in \mathbb{R}_{+}^{D}$ and $m \in \mathcal{D}(x)$, then

$$
m(\mathcal{E})=\lim _{n} m\left(\mathcal{E} \chi_{(\mathcal{E} \preceq n \mathbf{1})}\right) \leq\left\langle x, x^{\prime \prime}\right\rangle<\infty
$$

hence if $m \in \operatorname{dom}\left(\mathbb{U}_{0}^{*}\right)$ then

$$
\mathbb{U}_{\mathcal{E}}^{*}(m)=\mathbb{E}\left[\tilde{U}^{*}\left(\frac{d m^{c}}{d P}\right)\right]+m(\mathcal{E})<\infty .
$$

Define

$$
v_{\mathcal{E}}(x):=\inf _{m \in \mathcal{D}(x)}\left\{\mathbb{E}\left[\tilde{U}^{*}\left(\frac{d m^{c}}{d P}\right)\right]+m(\mathcal{E})\right\} .
$$

\footnotetext{
${ }^{\ddagger}$ We recall that a sequence of $\mathbb{R}^{D}$-valued random variables $X^{n}$ is Fatou-convergent to $\mathrm{X}$ if $X^{n} \rightarrow X$ a.s. and $X^{n}+a \mathbf{1} \in$ $L^{0}\left(K_{T}, \mathcal{F}_{T}\right)$ for some $a$. A set $\mathcal{A}_{0}$ is said to be Fatou-dense in $\mathcal{A}$ if any element of $\mathcal{A}$ is a limit of a Fatou-convergent sequence of elements from $\mathcal{A}_{0}$.
} 
We define, for $x \in \mathbb{R}_{+}^{D}, u_{\mathcal{E}}(x):=u(\mathcal{E}+x)$. Hence we have ${ }^{\S}$

$$
\begin{aligned}
u_{\mathcal{E}}(x) & =v_{\mathcal{E}+x}=\min _{m \in \mathcal{D} \cap \operatorname{dom}\left(\mathbb{U}_{0}^{*}\right)}\left\{\mathbb{E}\left[\tilde{U}^{*}\left(\frac{d m^{c}}{d P}\right)\right]+m(\mathcal{E}+x)\right\} \\
& =\inf _{y \in \mathbb{R}_{+}^{D}} \inf _{m \in \mathcal{D}(y) \cap \operatorname{dom}\left(\mathbb{U}_{0}^{*}\right)}\left\{\mathbb{E}\left[\tilde{U}^{*}\left(\frac{d m^{c}}{d P}\right)\right]+m(\mathcal{E})+\langle x, y\rangle\right\} \\
& =\min _{y \in \mathbb{R}_{+}^{D}}\left\{v_{\mathcal{E}}(y)+\langle x, y\rangle\right\}
\end{aligned}
$$

where the second equality is due to the fact that dom $\mathbb{U}_{0}^{*}=\operatorname{dom} \mathbb{U}_{\mathcal{E}}^{*}$ whenever $\mathcal{E} \in \mathcal{O}$ (see Remark 3.2). A consequence, $v_{\mathcal{E}}(y)$ is the convex conjugate of $u_{\mathcal{E}}(x)$, which implies $u_{\mathcal{E}}^{*}(y)=v_{\mathcal{E}}(y)$.

LEMMA 3.3 The infimum in (3.8) is attained whenever $v_{\mathcal{E}}(x)$ is finite.

Proof. Set $L^{\infty}=L^{\infty}\left(\mathbb{R}^{D}\right)$ and ba $=\mathrm{ba}\left(\mathbb{R}^{D}\right)$ for the sake of simplicity. Take $x \in \mathbb{R}_{+}^{D}$ such that $v_{\mathcal{E}}(x)$ is finite. We first show that $\mathcal{D}(x)$ is $\sigma\left(\mathrm{ba}, L^{\infty}\right)$-compact. To see this, remark first that the set $\mathcal{D}$ is $\sigma$ (ba, $L^{\infty}$ )-closed: for any sequence $\left(\mu_{n}\right)_{n \geq 0} \subseteq \mathcal{D}$ such that $\mu_{n} \rightarrow \mu$ in $\sigma\left(\right.$ ba, $\left.L^{\infty}\right)$ we also have $\mu(X)=\lim _{n} \mu_{n}(X) \leq 0$ for any $X \in \mathcal{C}$. To show closedness of $\mathcal{D}(x)$ take $\left(\mu_{n}\right)_{n \geq 0} \subseteq \mathcal{D}(x)$ such that $\mu_{n} \rightarrow \mu$ in $\sigma\left(\mathrm{ba}, L^{\infty}\right)$, then $\mu(\Omega)=\lim _{n} \mu_{n}(\Omega)=x$ and $\mu \in \mathcal{D}(x)$. The set $\mathcal{D}(x)$ only contains positive measures, for which $\|\mu\| \leq \mu(\Omega)$, hence it can be seen as a closed subset of the $\sigma\left(\mathrm{ba}, L^{\infty}\right)$-compact ball $\{\mu \in$ ba : $\|\mu\| \leq x\}$. Hence $\mathcal{D}(x)$ is $\sigma\left(\right.$ ba, $\left.L^{\infty}\right)$-compact.

It follows from basic properties of dual functions that $\mathbb{U}_{\mathcal{E}}^{*}(m)$ is $\sigma\left(\mathrm{ba}, L^{\infty}\right)$-lower semicontinuous (being the supremum of a sequence of affine functions). Then if $\left(\mu_{n}\right)_{n \geq 0} \subseteq \mathcal{D}(x)$ is a minimizing sequence in (3.8), we can extract a subsequence $\mu_{n_{k}}$ converging to $\mu$ in $\sigma$ (ba, $\left.L^{\infty}\right)$ as $k \rightarrow \infty$ and we have $\mathbb{U}_{\mathcal{E}}^{*}(\mu) \leq \liminf _{k} \mathbb{U}_{\mathcal{E}}^{*}\left(\mu_{n_{k}}\right)=\inf _{m \in \mathcal{D}(x)} \mathbb{U}_{\mathcal{E}}^{*}(m)$. Hence $\mathbb{U}_{\mathcal{E}}^{*}(\mu)=\inf _{m \in \mathcal{D}(x)} \mathbb{U}_{\mathcal{E}}^{*}(m)$ and $\mu$ attains the infimum in $(3.8)$.

\subsection{Existence of the optimizer}

Let $\mathcal{E} \in \mathcal{O}$. We now show that $v_{\mathcal{E}}: \mathbb{R}_{+}^{D} \rightarrow \mathbb{R}$ is a proper convex function. It is clearly proper by Proposition 3.1 (ii) and Lemma 3.1. Now, we turn to convexity. Let $m_{1}$ and $m_{2}$ be the minimizers in $v_{\mathcal{E}}\left(x_{1}\right)$ and $v_{\mathcal{E}}\left(x_{2}\right)$ and let $x=(1-\lambda) x_{1}+\lambda x_{2}, m=(1-\lambda) m_{1}+\lambda m_{2} \in \mathcal{D}(x) \cap \operatorname{dom}\left(\mathbb{U}_{0}^{*}\right)$. We have

$$
\begin{aligned}
& (1-\lambda) v_{\mathcal{E}}\left(x_{1}\right)+\lambda v_{\mathcal{E}}\left(x_{2}\right) \\
& =(1-\lambda)\left\{\mathbb{E}\left[\tilde{U}^{*}\left(\frac{d m_{1}^{c}}{d P}\right)\right]+m_{1}(\mathcal{E})\right\}+\lambda\left\{\mathbb{E}\left[\tilde{U}^{*}\left(\frac{d m_{2}^{c}}{d P}\right)\right]+m_{2}(\mathcal{E})\right\} \\
& =\mathbb{E}\left[(1-\lambda) \tilde{U}^{*}\left(\frac{d m_{1}^{c}}{d P}\right)+\lambda \tilde{U}^{*}\left(\frac{d m_{2}^{c}}{d P}\right)\right]+(1-\lambda) m_{1}(\mathcal{E})+\lambda m_{2}(\mathcal{E}) \\
& \geq \mathbb{E}\left[\tilde{U}^{*}\left((1-\lambda) \frac{d m_{1}^{c}}{d P}+\lambda \frac{d m_{2}^{c}}{d P}\right)\right]+(1-\lambda) m_{1}(\mathcal{E})+\lambda m_{2}(\mathcal{E}) \\
& =\mathbb{E}\left[\tilde{U}^{*}\left(\frac{d m^{c}}{d P}\right)\right]+m(\mathcal{E}) \geq v_{\mathcal{E}}(x) .
\end{aligned}
$$

Consider any $m \in \mathcal{D} \cap \operatorname{dom}\left(\mathbb{U}_{0}^{*}\right)$ and $m_{\lambda}:=\lambda m+(1-\lambda) \widehat{m} \in \mathcal{D} \cap \operatorname{dom}\left(\mathbb{U}_{0}^{*}\right)$ for $\lambda \in[0,1]$. The function

$$
h(\lambda)=\mathbb{E}\left[\tilde{U}^{*}\left(\frac{d m_{\lambda}^{c}}{d P}\right)\right]+m_{\lambda}(\mathcal{E})
$$

\footnotetext{
$\S_{\text {Let }} x \in \mathbb{R}_{+}^{D}$. Notice that, since $\mathbb{R}_{+}^{D} \subseteq \mathcal{I}:=\operatorname{int}\left(-\mathcal{A}_{T}^{0} \cap \mathbb{R}^{D}\right)$, one has that $x+x^{\prime} \in \mathcal{I}$ whenever $x^{\prime} \in \mathcal{I}$. This implies that if $\mathcal{E} \in \mathcal{O}$ and $x \in \mathbb{R}_{+}^{D}$, then $x+\mathcal{E}$ belongs to $\mathcal{O}$ and the duality representation (3.7) can be applied to $x+\mathcal{E}$ as well.
} 
is convex and has a minimum at zero, therefore by monotone convergence one has

$$
\begin{aligned}
0 & \leq h_{+}^{\prime}(0)=\lim _{\lambda \downarrow 0} \frac{h(\lambda)-h(0)}{\lambda} \\
& =\lim _{\lambda \downarrow 0}\left\{\mathbb{E}\left[\frac{\tilde{U}^{*}\left(\frac{d m_{\lambda}^{c}}{d P}\right)-\tilde{U}^{*}\left(\frac{d \widehat{m}^{c}}{d P}\right)}{\lambda}\right]+\frac{m_{\lambda}(\mathcal{E})-\widehat{m}(\mathcal{E})}{\lambda}\right\} \\
& =\mathbb{E}\left[\lim _{\lambda \downarrow 0} \frac{\tilde{U}^{*}\left(\frac{d m_{\lambda}^{c}}{d P}\right)-\tilde{U}^{*}\left(\frac{d \widehat{m}^{c}}{d P}\right)}{\lambda}\right]+m(\mathcal{E})-\widehat{m}(\mathcal{E}) \\
& =\mathbb{E}\left[\left\langle-\tilde{I}\left(\frac{d \widehat{m}^{c}}{d P}\right), \frac{d m^{c}}{d P}-\frac{d \widehat{m}^{c}}{d P}\right\rangle\right]+m(\mathcal{E})-\widehat{m}(\mathcal{E})
\end{aligned}
$$

so that

$$
\mathbb{E}\left[\left\langle\tilde{I}\left(\frac{d \widehat{m}^{c}}{d P}\right), \frac{d m^{c}}{d P}\right\rangle\right]-m(\mathcal{E}) \leq \mathbb{E}\left[\left\langle\tilde{I}\left(\frac{d \widehat{m}^{c}}{d P}\right), \frac{d \widehat{m}^{c}}{d P}\right\rangle\right]-\widehat{m}(\mathcal{E}) .
$$

Since $\mathbb{U}_{0}^{*}$ is decreasing with respect to the preorder induced by ba $\left(\mathbb{R}_{+}^{D}\right)$, if we take any $\tilde{m} \in \mathcal{D}$ we have that $m:=\widehat{m}+\tilde{m} \in \mathcal{D} \cap \operatorname{dom}\left(\mathbb{U}_{0}^{*}\right)$. It follows that

$$
\mathbb{E}\left[\left\langle\tilde{I}\left(\frac{d \widehat{m}^{c}}{d P}\right), \frac{d \tilde{m}^{c}}{d P}\right\rangle\right] \leq \tilde{m}(\mathcal{E}) .
$$

At this point, we would like to prove that we have equality in (3.9) when $\tilde{m}=\widehat{m}$. To do so, we need to impose an additional property to the value $u_{\mathcal{E}}(\cdot)$ which is the asymptotic satiability.

Assumption 3.2 Let $\mathcal{E} \in \mathcal{O} \cap L^{\infty}\left(\mathbb{R}_{+}^{D}\right)$. The function $u_{\mathcal{E}}: \mathbb{R}_{+}^{D} \rightarrow \mathbb{R}$ is asymptotically satiable.

Since $u_{\mathcal{E}}(\cdot)$ is asymptotically satiable, by Proposition 2.1 if $\mathcal{E} \in L^{\infty}$ there exists a $y \in \operatorname{dom}\left(u_{\mathcal{E}}^{*}\right)$ such that $\|\mathcal{E}\|_{\infty}\|y\|_{1} \leq \epsilon$ for any $\epsilon>0$, where $\|y\|_{1}=\sum_{i=1}^{D}\left|y_{i}\right|$. Also, by duality, there must exist an $m \in \mathcal{D}(y) \cap \operatorname{dom}\left(\mathbb{U}_{0}^{*}\right)$. Clearly $m(\mathcal{E}) \leq\|m\|_{\text {ba }\left(\mathbb{R}^{D}\right)}\|\mathcal{E}\|_{\infty} \leq \epsilon$ so that

$$
\begin{aligned}
-\epsilon \leq-m(\mathcal{E}) & \leq \mathbb{E}\left[\left\langle\tilde{I}\left(\frac{d \widehat{m}^{c}}{d P}\right), \frac{d m^{c}}{d P}\right\rangle\right]-m(\mathcal{E}) \\
& \leq \mathbb{E}\left[\left\langle\tilde{I}\left(\frac{d \widehat{m}^{c}}{d P}\right), \frac{d \widehat{m}^{c}}{d P}\right\rangle\right]-\widehat{m}(\mathcal{E}) \leq 0
\end{aligned}
$$

and, being $\epsilon$ arbitrary, this implies

$$
\mathbb{E}\left[\left\langle\tilde{I}\left(\frac{d \widehat{m}^{c}}{d P}\right), \frac{d \widehat{m}^{c}}{d P}\right\rangle\right]=\widehat{m}(\mathcal{E}) .
$$

Inequalities (3.9) and equality (3.10) allow us to prove the existence of the optimizer for the original maximization problem with random endowment $\mathcal{E} \in \mathcal{O}$, under the additional assumption that $\mathcal{E}$ is bounded.

THEOREM 3.2 Let $U: \mathbb{R}^{d} \rightarrow[-\infty, \infty)$ be a utility function supported on $\mathbb{R}_{+}^{d}$. Given any $\mathcal{E} \in \mathcal{O} \cap L^{\infty}$, if the value function verifies Assumption (3.2) then the optimal investment problem (3.2) has a unique solution $\widehat{X}:=\tilde{I}\left(\frac{d \widehat{m}^{c}}{d P}\right)-\mathcal{E}$, where $\widehat{m}$ is any optimizer in the dual problem.

Proof. Take any $Z \in \mathcal{Z}^{s}$ and let $m^{Z} \in \mathcal{D}$ be its corresponding measure as in Remark 3.3. It follows from (3.9) that $\mathbb{E}\left[\left\langle\widehat{X}+\mathcal{E}, Z_{T}\right\rangle\right]=\mathbb{E}\left[\left\langle\tilde{I}\left(\frac{d \widehat{m}^{c}}{d P}\right), \frac{d m^{Z}}{d P}\right\rangle\right] \leq m^{Z}(\mathcal{E})$, hence $\mathbb{E}\left[\left\langle\widehat{X}, \frac{d m^{Z}}{d P}\right\rangle\right] \leq 0$. It now follows from Theorem 2.1 that $\widehat{X} \in \mathcal{A}_{T}^{0}$. Hence by using (3.10) we can write

$$
\begin{aligned}
\mathbb{E}[\tilde{U}(\widehat{X}+\mathcal{E})] & =\mathbb{E}\left[\tilde{U}\left(\tilde{I}\left(\frac{d \widehat{m}^{c}}{d P}\right)\right)\right]=\mathbb{E}\left[\tilde{U}^{*}\left(\frac{d \widehat{m}^{c}}{d P}\right)\right]+\mathbb{E}\left[\left\langle\tilde{I}\left(\frac{d \widehat{m}^{c}}{d P}\right), \frac{d \widehat{m}^{c}}{d P}\right\rangle\right] \\
& =\mathbb{E}\left[\tilde{U}^{*}\left(\frac{d \widehat{m}^{c}}{d P}\right)\right]+\widehat{m}(\mathcal{E})=\mathbb{U}_{\mathcal{E}}^{*}(\widehat{m}) .
\end{aligned}
$$

It is now easy to conclude by using Theorem 3.1. Uniqueness follows by the same arguments used in [CO10, Theorem 3.12]. 
REMARK 3.4 It is important to stress that the boundedness assumption on the random endowment $\mathcal{E}$ is needed only to prove the existence of the optimal portfolio, while to obtain the duality characterization and the existence of the minimizer in the dual problem it suffices to require the weaker property $\mathcal{E} \in \mathcal{O}$, i.e. the random endowment can be unbounded from above.

\subsection{Sufficient conditions for existence and liquidation}

We can now give some conditions which ensure asymptotic satiability of $u_{\mathcal{E}}(x)$. In order to check them easily, it is useful, in general, to look for conditions that only concern the utility function $U$ (or possibly its dual). We start by defining a growth condition in the version of [CO10] (even if similar conditions have appeared in different papers, for example in [DPT01]).

Definition 3.1 Let $U: \mathbb{R}^{d} \rightarrow[-\infty, \infty)$ be a utility function. We shall say that the dual function $U^{*}$ satisfies the growth condition if there exists a function $\zeta:(0,1] \rightarrow[0, \infty)$ such that for all $\epsilon \in(0,1]$ and all $x^{*} \in \operatorname{int}\left(\mathbb{R}_{+}^{d}\right)$

$$
U^{*}\left(\epsilon x^{*}\right) \leq \zeta(\epsilon)\left(U^{*}\left(x^{*}\right)^{+}+1\right) .
$$

The following result is the analogue of [CO10, Corollary 3.7]. The proof is essentially the same with some minor modifications. Nonetheless, we decided to give the details for reader's convenience.

LEMma 3.4 Take $\mathcal{E} \in \mathcal{O} \cap L^{\infty}\left(\mathbb{R}^{D}\right)$. If $U^{*}$ satisfies the growth condition (3.11) then both $U$ and $u_{\mathcal{E}}(\cdot)$ are asymptotically satiable.

Proof. Take $m \in \mathcal{D} \cap \operatorname{dom}\left(\mathbb{U}_{0}^{*}\right)$ (for example the minimizer in the dual problem $(3.7)$ ), define $x^{*}:=m(\Omega$ ). Then, since $\mathcal{E} \preceq x^{\prime \prime}+X^{\prime \prime}$ with $x^{\prime \prime} \in \mathcal{I}$ and $X^{\prime \prime} \in \mathcal{C}$, one has

$$
\begin{aligned}
u_{\mathcal{E}}^{*}\left(\epsilon x^{*}\right) & =v_{\mathcal{E}}\left(\epsilon x^{*}\right) \leq \mathbb{E}\left[\tilde{U}^{*}\left(\epsilon \frac{d m^{c}}{d P}\right)\right]+\epsilon m(\mathcal{E}) \leq \mathbb{E}\left[U^{*}\left(\epsilon P\left(\frac{d m^{c}}{d P}\right)\right)\right]+\epsilon\left\langle x^{*}, x^{\prime \prime}\right\rangle \\
& \leq \zeta(\epsilon) \mathbb{E}\left[U^{*}\left(P\left(\frac{d m^{c}}{d P}\right)\right)^{+}+1\right]+\epsilon\left\langle x^{*}, x^{\prime \prime}\right\rangle=\zeta(\epsilon) \mathbb{E}\left[\tilde{U}^{*}\left(\frac{d m^{c}}{d P}\right)^{+}+1\right]+\epsilon\left\langle x^{*}, x^{\prime \prime}\right\rangle\langle\infty
\end{aligned}
$$

for any $\epsilon \in(0,1]$. Hence $\epsilon x^{*} \in \operatorname{dom}\left(u_{\mathcal{E}}^{*}\right)$. Taking the limit as $\epsilon \rightarrow 0$ shows that $0 \in \operatorname{cl}\left(\operatorname{dom}\left(u_{\mathcal{E}}^{*}\right)\right)$ and hence $u_{\mathcal{E}}$ is asymptotically satiable by Proposition 2.1. The proof for $U$ follows the same lines but in an easier way, by directly using the growth condition and the characterization of Proposition 2.1 as in [CO10, Corollary 3.7].

One might look for sufficient conditions to check that the growth condition (3.11) actually holds. In [CO10] the notion of reasonable asymptotic elasticity of $U$ is introduced in order to ensure the growth condition in the case of multivariate utility functions which are multivariate risk-averse and bounded from below. If $U$ is bounded from above then (3.11) trivially holds with $\zeta(\epsilon):=\sup _{x^{*} \in \mathbb{R}_{+}^{d}} U^{*}\left(x^{*}\right)=$ $U^{*}(0)=\sup _{x \in \mathbb{R}^{d}} U(x)<\infty$. It is also satisfied if the quantity $-\left\langle\nabla U^{*}\left(\epsilon x^{*}\right), x^{*}\right\rangle$ is bounded from above in $x^{*}$ (as in the case of the sum of logarithms, a utility function which is neither bounded from above nor from below).

REMARK 3.5 Some papers dealing with optimal investment assume that the agent liquidates his assets at the terminal date to one (ore more) reference assets. As in [CO10], it is possible to show that the problem treated here is essentially equivalent to the investment problem with final liquidation, provided that $U$ is upper semi-continuous. In particular we have that

$$
u(\mathcal{E})=\sup _{W \in \mathcal{A}_{T-}^{0}} \mathbb{E}[\bar{U}(W+\mathcal{E})]
$$

where

$$
\bar{U}(W):=\sup \left\{U(\xi): \xi \in \mathbb{R}_{+}^{d},(\xi, \underline{0})-W \in-K_{T}\right\}, \quad W \in L^{0}\left(K_{T}, \mathscr{F}_{T-}\right)
$$

and $\underline{0}$ denotes the zero vector in $\mathbb{R}^{D-d}$. The proof follows the same lines as [CO10], Proposition 4.3 with minor modifications.

If $\mathcal{E} \in \mathcal{O} \cap L^{\infty}$ (which ensures the existence of a solution in the primal problem) then we can argue as in [CO10, Proposition 4.4] to conclude that the supremum in (3.12) is attained at some $\widehat{W} \in \mathcal{A}_{T-}^{0}$ and that $(\widehat{\xi}(\widehat{W}+\mathcal{E}), \underline{0})=\widehat{X}+\mathcal{E}$ a.s., where $\widehat{\xi}(\widehat{W}+\mathcal{E})$ is the maximizer in $(3.13)$. 
REMARK 3.6 If $d=1$ our optimization problem is similar to that treated in [Bou02]. In that paper, however, the utility function is defined on the whole real line, which permits to avoid recurring to singular measures. In a sense, we generalize their results in that we do not require the underlying asset processes to be continuous, nor the transaction costs to be constant (we work in the framework set out in [CS06] which is much more general). Moreover, we allow for a liquidation to many assets, which forces us to introduce multivariate utility functions. Finally, many of our results (e.g. the duality characterization) do not require the boundedness of the endowment which is instead assumed in [Bou02].

\section{Utility indifference pricing}

In this section we will examine some applications of the above results to the pricing of contingent claims in an incomplete market. The analysis that follows is motivated by the fast growing interest in new pricing paradigms (alternative to replication) in the context of incomplete financial markets. We adopt some of the techniques used in [OZ09], where the authors studied a similar investment problem but in a framework of frictionless financial markets and with univariate utility functions (defined on the whole real line).

We start by proving some continuity properties of the value function.

LEMMA 4.1 (i) If $\left(\mathcal{E}_{n}\right)_{n \in \mathbb{N}}$ is a sequence of endowments in $\mathcal{O}$ and such that

$$
\sup _{m \in \mathcal{D} \cap \operatorname{dom}\left(\mathbb{U}_{0}^{*}\right)} m\left(\mathcal{E}_{n}-\mathcal{E}\right) \rightarrow 0 \text { and } \inf _{m \in \mathcal{D} \cap \operatorname{dom}\left(\mathbb{U}_{0}^{*}\right)} m\left(\mathcal{E}_{n}-\mathcal{E}\right) \rightarrow 0
$$

as $n \rightarrow \infty$ with $\mathcal{E} \in \mathcal{O}$, then $u\left(\mathcal{E}_{n}\right) \rightarrow u(\mathcal{E})$.

(ii) If $U$ is lower semi-continuous then $u$ is as well on $\mathcal{O}$ equipped with the topology of convergence in probability.

(iii) If $\left(x_{n}+\mathcal{E}\right)_{n \in \mathbb{N}} \in \mathcal{O}$ and $\left(x_{n}\right)_{n \in \mathbb{N}}$ is a sequence in $\mathbb{R}^{D}$ such that $x_{n} \rightarrow x$ and $a \preceq x_{n} \preceq b$ for some $a, b \in \mathbb{R}^{D}$, then $x+\mathcal{E} \in \mathcal{O}$ and

$$
u(\mathcal{E}+x)=\lim _{n} u\left(\mathcal{E}+x_{n}\right)
$$

(iv) If $\left(\mathcal{E}_{n}\right)_{n \in \mathbb{N}}$ is a sequence of endowments in $\mathcal{O} \cap L^{\infty}\left(\mathbb{R}^{D}\right)$ which uniformly satisfy equation (3.1) (in the sense that the upper and lower bounds do not depend on $n)$ and such that $\mathcal{E}_{n} \rightarrow \mathcal{E}$ in $L^{\infty}\left(\mathbb{R}^{D}\right)$ then we have

$$
u(\mathcal{E})=\lim _{n} u\left(\mathcal{E}_{n}\right)
$$

Proof. (i) We have

$$
\begin{aligned}
u\left(\mathcal{E}_{n}\right)-u(\mathcal{E})= & \inf _{m \in \mathcal{D} \cap \operatorname{dom}\left(\mathbb{U}_{0}^{*}\right)}\left\{\mathbb{E}\left[\tilde{U}^{*}\left(\frac{d m^{c}}{d P}\right)\right]+m\left(\mathcal{E}_{n}\right)\right\} \\
& -\inf _{m \in \mathcal{D} \cap \operatorname{dom}\left(\mathbb{U}_{0}^{*}\right)}\left\{\mathbb{E}\left[\tilde{U}^{*}\left(\frac{d m^{c}}{d P}\right)\right]+m(\mathcal{E})\right\} \\
& \leq \sup _{m \in \mathcal{D} \cap \operatorname{dom}\left(\mathbb{U}_{0}^{*}\right)} m\left(\mathcal{E}_{n}-\mathcal{E}\right)
\end{aligned}
$$

but also

$$
u\left(\mathcal{E}_{n}\right)-u(\mathcal{E}) \geq \inf _{m \in \mathcal{D} \cap \operatorname{dom}\left(\mathbb{U}_{0}^{*}\right)} m\left(\mathcal{E}_{n}-\mathcal{E}\right)
$$

hence $\left|u\left(\mathcal{E}_{n}\right)-u(\mathcal{E})\right| \rightarrow 0$ as $n \rightarrow \infty$.

(ii) Let $\left(\mathcal{E}_{n}\right)_{n \in \mathbb{N}}$ be a sequence of endowments in $\mathcal{O}$ such that $\mathcal{E}_{n} \rightarrow \mathcal{E}$ in probability $(\mathcal{E} \in \mathcal{O})$. Then a subsequence (that we still call in the same way) converges a.s. and we have, by semi-continuity of $U$ and Fatou's lemma

$$
\begin{aligned}
u\left(\liminf _{n} \mathcal{E}_{n}\right) & =\sup _{X \in \mathcal{A}_{T}^{0}} \mathbb{E}\left[\tilde{U}\left(X+\liminf _{n} \mathcal{E}_{n}\right)\right] \leq \sup _{X \in \mathcal{A}_{T}^{0}} \mathbb{E}\left[\liminf _{n} \tilde{U}\left(X+\mathcal{E}_{n}\right)\right] \\
& \leq \sup _{X \in \mathcal{A}_{T}^{0}} \liminf _{n} \mathbb{E}\left[\tilde{U}\left(X+\mathcal{E}_{n}\right)\right] \leq \liminf _{n} \sup _{X \in \mathcal{A}_{T}^{0}} \mathbb{E}\left[\tilde{U}\left(X+\mathcal{E}_{n}\right)\right]=\liminf _{n} u\left(\mathcal{E}_{n}\right)
\end{aligned}
$$


which implies the claim.

(iii) Let $\left(x_{n}+\mathcal{E}\right)_{n \in \mathbb{N}} \in \mathcal{O}$ with $\left(x_{n}\right)_{n \in \mathbb{N}}$ a sequence in $\mathbb{R}^{D}$ such that $x_{n} \rightarrow x$ and $a \preceq x_{n} \preceq b$ for some $a, b \in \mathbb{R}^{D}$, then $x+\mathcal{E} \in \mathcal{O}$ (since $-\mathcal{A}_{T}^{0} \cap \mathbb{R}^{D}$ is closed) and we have

$$
\begin{aligned}
u(\mathcal{E}+x) & =\inf _{m \in \mathcal{D} \cap \operatorname{dom}\left(\mathbb{U}_{0}^{*}\right)}\left\{\mathbb{E}\left[\tilde{U}^{*}\left(\frac{d m^{c}}{d P}\right)\right]+m(\mathcal{E})+m\left(\limsup x_{n}\right)\right\} \\
& =\inf _{m \in \mathcal{D} \cap \operatorname{dom}\left(\mathbb{U}_{0}^{*}\right)}\left\{\mathbb{E}\left[\tilde{U}^{*}\left(\frac{d m^{c}}{d P}\right)\right]+m(\mathcal{E})+\underset{n}{\left.\limsup m\left(x_{n}\right)\right\}}\right. \\
& \geq \limsup _{n} \inf _{m \in \mathcal{D} \cap \operatorname{dom}\left(\mathbb{U}_{0}^{*}\right)}\left\{\mathbb{E}\left[\tilde{U}^{*}\left(\frac{d m^{c}}{d P}\right)\right]+m\left(\mathcal{E}+x_{n}\right)\right\} \\
& =\limsup _{n} u\left(\mathcal{E}+x_{n}\right)
\end{aligned}
$$

hence $u$ is continuous along such sequences.

(iv) Let $\left(\mathcal{E}_{n}\right)_{n \in \mathbb{N}}$ be a sequence of endowments in $\mathcal{O} \cap L^{\infty}\left(\mathbb{R}^{D}\right)$ which uniformly satisfy equation (3.1) and such that $\mathcal{E}_{n} \rightarrow \mathcal{E}$ in $L^{\infty}\left(\mathbb{R}^{D}\right)$. Thus, we have

$$
\begin{aligned}
u(\mathcal{E}) & =\inf _{m \in \mathcal{D} \cap \operatorname{dom}\left(\mathbb{U}_{0}^{*}\right)}\left\{\mathbb{E}\left[\tilde{U}^{*}\left(\frac{d m^{c}}{d P}\right)\right]+m\left(\lim _{n} \mathcal{E}_{n}\right)\right\} \\
& =\inf _{m \in \mathcal{D} \cap \operatorname{dom}\left(\mathbb{U}_{0}^{*}\right)}\left\{\mathbb{E}\left[\tilde{U}^{*}\left(\frac{d m^{c}}{d P}\right)\right]+\lim _{n} m\left(\mathcal{E}_{n}\right)\right\} \\
& \geq \limsup _{n} \inf _{m \in \mathcal{D} \cap \operatorname{dom}\left(\mathbb{U}_{0}^{*}\right)}\left\{\mathbb{E}\left[\tilde{U}^{*}\left(\frac{d m^{c}}{d P}\right)\right]+m\left(\mathcal{E}_{n}\right)\right\} \\
& =\limsup _{n} u\left(\mathcal{E}_{n}\right)
\end{aligned}
$$

since $\mathcal{E}_{n} \rightarrow \mathcal{E}$ in $\sigma\left(L^{\infty}\left(\mathbb{R}^{D}\right)\right.$, ba $\left.\left(\mathbb{R}^{D}\right)\right)$. Hence $u$ is continuous also along these sequences as well.

For $j=1, \ldots, d$ define

$$
m_{j}(X):=\sum_{i=1}^{D} \int_{\Omega} X_{i} \frac{d m_{i}}{m_{j}(\Omega)}
$$

and

$$
\underline{m}_{j}(X):=\inf _{m \in \mathcal{D} \cap \operatorname{dom}\left(\mathbb{U}_{0}^{*}\right)}\left\{\sum_{i=1}^{D} \int_{\Omega} X_{i} \frac{d m_{i}}{m_{j}(\Omega)}\right\}
$$

For $B \in L^{0}\left(\mathbb{R}_{+}^{D}\right)$ denote $u_{\mathcal{E}}(B):=u(\mathcal{E}+B)$ (sometimes we will write $u_{\mathcal{E}}$ instead of $u(\mathcal{E})$ ). The following lemma will be useful for the characterization of utility indifference prices, which will be introduced immediately after.

Lemma 4.2 If $\mathcal{E} \in \mathcal{O}$ and $\mathcal{E}+B-e_{j} \widehat{m}_{j}(B) \in \mathcal{O}$ then

$$
u_{\mathcal{E}}\left(B-e_{j} \widehat{m}_{j}(B)\right) \leq u_{\mathcal{E}} \leq u_{\mathcal{E}}\left(B-e_{j} \underline{m}_{j}(B)\right)
$$

for all $j=1, \ldots, d$.

Proof. Remark first that the conditions above imply also that $\mathcal{E}+B-e_{j} \underline{m}_{j}(B) \in \mathcal{O}$. Using the duality characterization in Theorem 3.1 together with the definitions of $m_{j}$ and $\underline{m}_{j}$ yields

$$
\begin{aligned}
& u_{\mathcal{E}}\left(B-e_{j} \underline{m}_{j}(B)\right)= \\
& =\inf _{m \in \mathcal{D} \cap \operatorname{dom}\left(\mathbb{U}_{0}^{*}\right)}\left\{\mathbb{E}\left[\tilde{U}^{*}\left(\frac{d m^{c}}{d P}\right)\right]+m(\mathcal{E})+m\left(B-e_{j} \underline{m}_{j}(B)\right)\right\} \\
& \geq \inf _{m \in \mathcal{D} \cap \operatorname{dom}\left(\mathbb{U}_{0}^{*}\right)}\left\{\mathbb{E}\left[\tilde{U}^{*}\left(\frac{d m^{c}}{d P}\right)\right]+m(\mathcal{E})\right\}+\inf _{m \in \mathcal{D} \cap \operatorname{dom}\left(\mathbb{U}_{0}^{*}\right)}\left\{m\left(B-e_{j} \underline{m}_{j}(B)\right)\right\} \\
& =\inf _{m \in \mathcal{D} \cap \operatorname{dom}\left(\mathbb{U}_{0}^{*}\right)}\left\{\mathbb{E}\left[\tilde{U}^{*}\left(\frac{d m^{c}}{d P}\right)\right]+m(\mathcal{E})\right\}+\inf _{m \in \mathcal{D} \cap \operatorname{dom}\left(\mathbb{U}_{0}^{*}\right)}\left\{\left(\sum_{i=1}^{D} \int_{\Omega} B_{i} \frac{d m_{i}}{m_{j}(\Omega)}-\underline{m}_{j}(B)\right) m_{j}(\Omega)\right\} \\
& =v_{\mathcal{E}}+0=u_{\mathcal{E}} .
\end{aligned}
$$


On the other hand

$$
\begin{aligned}
& u_{\mathcal{E}}\left(B-e_{j} \widehat{m}_{j}(B)\right)= \\
& =\inf _{m \in \mathcal{D} \cap \operatorname{dom}\left(\mathbb{U}_{0}^{*}\right)}\left\{\mathbb{E}\left[\tilde{U}^{*}\left(\frac{d m^{c}}{d P}\right)\right]+m(\mathcal{E})+m\left(B-e_{j} \widehat{m}_{j}(B)\right)\right\} \\
& \leq \mathbb{E}\left[\tilde{U}^{*}\left(\frac{d \widehat{m}^{c}}{d P}\right)\right]+\widehat{m}(\mathcal{E})+\widehat{m}\left(B-e_{j} \widehat{m}_{j}(B)\right)=\mathbb{E}\left[\tilde{U}^{*}\left(\frac{d \widehat{m}^{c}}{d P}\right)\right]+\widehat{m}(\mathcal{E})+0=u_{\mathcal{E}} .
\end{aligned}
$$

which yields the other inequality.

Definition 4.1 For $j=1, \ldots, d$ the utility indifference (bid) price $(\mathrm{UIP}) p_{j}(B)=p_{j}(B ; U, \mathcal{E}) \in \mathbb{R}$ for the contingent claim $B$ (expressed in units of asset $j$ ) is implicitly defined as the solution to the equation

$$
u\left(\mathcal{E}+B-e_{j} p_{j}\right)=u(\mathcal{E})
$$

In the next proposition we show that the definition of UIP is well-posed, i.e. $p_{j}(B)$ exists unique, and that it satisfies in particular the properties of cash-invariance, monotonicity and convexity characterizing a convex risk measure defined on vector-valued random variables (compare [BR06, JMT, HHR10]).

Proposition 4.1 Let $j=1, \ldots, d$. Under the assumptions of Lemma 4.2 there exists a unique solution to (4.1). The UIP $p_{j}(B)$ is therefore well defined and it verifies the following properties:

(i) $\underline{m}_{j}(B) \leq p_{j}(B) \leq \widehat{m}_{j}(B)$;

(ii) if $B \in \mathcal{A}_{T}^{0}$ then $p_{j}(B) \leq 0$ for any $j=1, \ldots, d$;

(iii) for $c \in \mathbb{R}$ we have $p_{j}\left(B+e_{j} c\right)=p_{j}(B)+c$;

(iv) if $B \preceq C$ then $p_{j}(B) \leq p_{j}(C)$ for any $j=1, \ldots, d$;

(v) given contingent claims $B_{1}, B_{2}$ and $\lambda \in[0,1]$

$$
p_{j}\left(\lambda B_{1}+(1-\lambda) B_{2}\right) \geq \lambda p_{j}\left(B_{1}\right)+(1-\lambda) p_{j}\left(B_{2}\right)
$$

for any $j=1, \ldots, d$;

(vi) the utility indifference price can be expressed as

$$
p_{j}(B)=\inf _{m \in \mathcal{D}^{j}(1) \cap \operatorname{dom}\left(\mathbb{U}_{0}^{*}\right)}\left\{m_{j}(B)+\alpha_{j}(m)\right\}
$$

where

$$
\begin{gathered}
\mathcal{D}^{j}(k):=\left\{m \in \mathcal{D}: m_{j}(\Omega)=k\right\} \\
\alpha_{j}(m):=\inf _{k>0} \frac{1}{k}\left\{\mathbb{E}\left[\tilde{U}^{*}\left(\frac{d m^{k, c}}{d P}\right)\right]+m^{k}(\mathcal{E})-v_{\mathcal{E}}\right\}
\end{gathered}
$$

and $m^{k}$ is such that $m_{i}^{k}=m_{i}$ if $i \neq j$ and $m_{j}^{k}=k m_{j}$;

(vii) if $\left(B_{n}\right)_{n \in \mathbb{N}}$ is a sequence of contingent claims such that

$$
\inf _{m \in \mathcal{D}^{j}(1) \cap \operatorname{dom}\left(\mathbb{U}_{0}^{*}\right)} m_{j}\left(B_{n}-B\right) \rightarrow 0 \text { and } \sup _{m \in \mathcal{D}^{j}(1) \cap \operatorname{dom}\left(\mathbb{U}_{0}^{*}\right)} m_{j}\left(B_{n}-B\right) \rightarrow 0
$$

then $p_{j}\left(B_{n}\right) \rightarrow p_{j}(B)$.

Proof. Remark first that if $\mathcal{E}_{1}$ belongs to $\mathcal{O}$ and $\mathcal{E}_{2}:=\mathcal{E}_{1}+x$ with $x \in \mathbb{R}_{+}^{D}$ and $x_{j}>0$ for some $j \leq d$, then $u\left(\mathcal{E}_{2}\right)>u\left(\mathcal{E}_{1}\right)$. Indeed we have

$$
\begin{aligned}
u\left(\mathcal{E}_{1}\right) & =\mathbb{E}\left[\tilde{U}^{*}\left(\frac{d \widehat{m}_{1}^{c}}{d P}\right)\right]+\widehat{m}_{1}\left(\mathcal{E}_{1}\right) \leq \mathbb{E}\left[\tilde{U}^{*}\left(\frac{d \widehat{m}_{2}^{c}}{d P}\right)\right]+\widehat{m}_{2}\left(\mathcal{E}_{2}-x\right) \\
& <\mathbb{E}\left[\tilde{U}^{*}\left(\frac{d \widehat{m}_{2}^{c}}{d P}\right)\right]+\widehat{m}_{2}\left(\mathcal{E}_{2}\right)=u\left(\mathcal{E}_{2}\right)
\end{aligned}
$$


where $\widehat{m}_{1}$ (resp. $\widehat{m}_{2}$ ) is the minimizer in the dual problem with endowment $\mathcal{E}_{1}$ (resp. $\mathcal{E}_{2}$ ).

Existence and uniqueness follow from Lemma 4.1(iii) and the above considerations. Property (i) is clear from Lemma 4.2. Property (ii) follows from the definition of the primal problem by noting that $X+B \in \mathcal{A}_{T}^{0}$ if $X, B \in \mathcal{A}_{T}^{0}$. In particular we have $u(\mathcal{E}+B) \leq u(\mathcal{E})=u\left(\mathcal{E}+B-e_{j} p_{j}\right)$ which implies the claim. Property (iii) is straightforward from the definition of UIP and (iv) follows by monotonicity of $u_{\mathcal{E}}(\cdot)$.

(v) By concavity of $u_{\mathcal{E}}(\cdot)$

$$
\begin{aligned}
u_{\mathcal{E}}\left(\lambda B_{1}+\right. & \left.(1-\lambda) B_{2}-e_{j} \lambda p_{j}\left(B_{1}\right)-e_{j}(1-\lambda) p_{j}\left(B_{2}\right)\right) \\
& \geq \lambda u_{\mathcal{E}}\left(B_{1}-e_{j} p_{j}\left(B_{1}\right)\right)+(1-\lambda) u_{\mathcal{E}}\left(B_{2}-e_{j} p_{j}\left(B_{2}\right)\right)=u_{\mathcal{E}} \\
& =u_{\mathcal{E}}\left(\lambda B_{1}+(1-\lambda) B_{2}-e_{j} p_{j}\left(\lambda B_{1}+(1-\lambda) B_{2}\right)\right)
\end{aligned}
$$

by definition of UIP. The claim follows by monotonicity of $u_{\mathcal{E}}(\cdot)$.

(vi) By monotonicity of $u_{\mathcal{E}}(\cdot)$ and Lemma 4.1(iii), we have

$$
\begin{aligned}
p_{j}(B) & =\inf \left\{p: u_{\mathcal{E}}\left(B-e_{j} p\right)<v_{\mathcal{E}}\right\} \\
& =\inf \left\{p: \inf _{m \in \mathcal{D} \cap \operatorname{dom}\left(\mathbb{U}_{0}^{*}\right)}\left\{\mathbb{E}\left[\tilde{U}^{*}\left(\frac{d m^{c}}{d P}\right)\right]+m(\mathcal{E})+m(B)-p m_{j}(\Omega)\right\}<v_{\mathcal{E}}\right\} \\
& =\inf \left\{p: \inf _{m \in \mathcal{D}^{j}(1) \cap \operatorname{dom}\left(\mathbb{U}_{0}^{*}\right)} \inf _{k>0}\left\{\mathbb{E}\left[\tilde{U}^{*}\left(\frac{d m^{k, c}}{d P}\right)\right]+m^{k}(\mathcal{E})+k m_{j}(B)-v_{\mathcal{E}}-k p\right\}<0\right\} \\
& =\inf \left\{p: \inf _{m \in \mathcal{D}^{j}(1) \cap \operatorname{nom}\left(\mathbb{U}_{0}^{*}\right)} m_{j}(B)+\inf _{k>0} \frac{1}{k}\left\{\mathbb{E}\left[\tilde{U}^{*}\left(\frac{d m^{k, c}}{d P}\right)\right]+m^{k}(\mathcal{E})-v_{\mathcal{E}}\right\}<p\right\} \\
& =\inf _{m \in \mathcal{D}^{j}(1) \cap \operatorname{dom}\left(\mathbb{U}_{0}^{*}\right)}\left\{m_{j}(B)+\inf _{k>0} \frac{1}{k}\left\{\mathbb{E}\left[\tilde{U}^{*}\left(\frac{d m^{k, c}}{d P}\right)\right]+m^{k}(\mathcal{E})-v_{\mathcal{E}}\right\}\right\} \\
& =\inf _{m \in \mathcal{D}^{j}(1) \cap \operatorname{dom}\left(\mathbb{U}_{0}^{*}\right)}\left\{m_{j}(B)+\alpha_{j}(m)\right\}
\end{aligned}
$$

where we recall that

$$
\begin{gathered}
\mathcal{D}^{j}(k):=\left\{m \in \mathcal{D}: m_{j}(\Omega)=k\right\} \\
\alpha_{j}(m):=\inf _{k>0} \frac{1}{k}\left\{\mathbb{E}\left[\tilde{U}^{*}\left(\frac{d m^{k, c}}{d P}\right)\right]+m^{k}(\mathcal{E})-v_{\mathcal{E}}\right\}
\end{gathered}
$$

and $m^{k}$ is such that $m_{i}^{k}=m_{i}$ if $i \neq j$ and $m_{j}^{k}=k m_{j}$.

(vii) Remark that

$$
\begin{aligned}
\inf _{m \in \mathcal{D}^{j}(1) \cap \operatorname{dom}\left(\mathbb{U}_{0}^{*}\right)} & m_{j}\left(B_{n}-B\right)=\inf _{m \in \mathcal{D}^{j}(1) \cap \operatorname{dom}\left(\mathbb{U}_{0}^{*}\right)}\left[\left(m_{j}\left(B_{n}\right)+\alpha_{j}(m)\right)-\left(m_{j}(B)+\alpha_{j}(m)\right)\right] \\
& \leq \inf _{m \in \mathcal{D}^{j}(1) \cap \operatorname{dom}\left(\mathbb{U}_{0}^{*}\right)}\left[m_{j}\left(B_{n}\right)+\alpha_{j}(m)\right]-\inf _{m \in \mathcal{D}^{j}(1) \cap \operatorname{dom}\left(\mathbb{U}_{0}^{*}\right)}\left[m_{j}(B)+\alpha_{j}(m)\right] \\
& =p_{j}\left(B_{n}\right)-p_{j}(B) \leq \sup _{m \in \mathcal{D}^{j}(1) \cap \operatorname{dom}\left(\mathbb{U}_{0}^{*}\right)} m_{j}\left(B_{n}-B\right)
\end{aligned}
$$

which implies the claim.

Definition 4.2 The average utility indifferent purchase price for $\beta$ units of the contingent claim $B$ (in terms of asset $j$ ) is defined by

$$
p_{j}^{\beta}(B):=\frac{p_{j}(\beta B)}{\beta} .
$$

In the next proposition we present some properties of the function $\beta \mapsto p_{j}^{\beta}(B)$.

Proposition 4.2 If $\mathcal{E} \in \mathcal{O}$ and $\mathcal{E}+\beta\left(B-e_{j} \widehat{m}_{j}(B)\right) \in \mathcal{O}$ for all $\beta>0$ then the function $\beta \mapsto p_{j}^{\beta}(B)$ verifies the following properties:

(i) It is non-increasing in $\beta$;

(ii) $\underline{m}_{j}(B) \leq p_{j}^{\beta}(B) \leq \widehat{m}_{j}(B)$ for all $\beta>0$;

(iii) $\lim _{\beta \rightarrow \infty} p_{j}^{\beta}(B)=\underline{m}_{j}(B)$; 
Proof. Remark first that the conditions of Lemma 4.2 are automatically satisfied by $\beta B$ for all $\beta>0$.

(i) Take $0<\beta_{1} \leq \beta_{2}$. Then by concavity (Proposition $4.1(\mathrm{v})$ ) we have

$$
p_{j}^{\beta_{1}}(B):=\frac{1}{\beta_{1}} p_{j}\left(\frac{\beta_{1}}{\beta_{2}} \beta_{2} B\right) \geq \frac{1}{\beta_{2}} p_{j}\left(\beta_{2} B\right)+\left(\frac{1}{\beta_{1}}-\frac{1}{\beta_{2}}\right) p_{j}(0)=\frac{1}{\beta_{2}} p_{j}\left(\beta_{2} B\right)=p_{j}^{\beta_{2}}(B) .
$$

(ii) It is clear from Proposition 4.1.

(iii) Suppose for a contradiction that there exists $\tilde{m} \in \mathcal{D} \cap \operatorname{dom}\left(\mathbb{U}_{0}^{*}\right)$ such that $\tilde{m}_{j}(B)<\lim _{\beta \rightarrow \infty} p_{j}^{\beta}(B)$. Then for any $\beta>0$

$$
\begin{aligned}
u_{\mathcal{E}} & =u_{\mathcal{E}}\left(\beta B-e_{j} p_{j}(\beta B)\right)=\inf _{m \in \mathcal{D} \cap \operatorname{dom}\left(\mathbb{U}_{0}^{*}\right)}\left\{\mathbb{E}\left[\tilde{U}^{*}\left(\frac{d m^{c}}{d P}\right)\right]+m(\mathcal{E})+\beta m\left(B-e_{j} p_{j}^{\beta}(B)\right)\right\} \\
& \leq \mathbb{E}\left[\tilde{U}^{*}\left(\frac{d \tilde{m}^{c}}{d P}\right)\right]+\tilde{m}(\mathcal{E})+\beta \tilde{m}_{j}(\Omega)\left[\tilde{m}_{j}(B)-p_{j}^{\beta}(B)\right]
\end{aligned}
$$

and we get the desired contradiction by sending $\beta$ to infinity.

Let us look for an interpretation of the previous result. Assume the agent has purchased the claim $B$ paying $p$ units of asset $j$ and now wants to eliminate all the risk arising from this position by superhedging the claim $-B$. By Theorem 2.1 he will be able to reach his objective if and only if

$$
\inf _{Z \in \mathcal{Z}^{s}} \mathbb{E}\left[\left\langle B, \frac{Z_{T}}{Z_{0}^{j}}\right\rangle\right] \geq p
$$

hence the highest price he will accept to pay for the claim $B$ (in units of asset $j$ ) by remaining sure that he will run no risks at maturity is

$$
\bar{p}_{j}(B):=\inf _{Z \in \mathcal{Z}^{s}} \mathbb{E}\left[\left\langle B, \frac{Z_{T}}{Z_{0}^{j}}\right\rangle\right]
$$

Now suppose that $B$ is bounded, which is the case for most common claims like call and put options (recall that we are working with units and not with prices). Thus (by definition of $\bar{p}_{j}(B)$ and Theorem 2.1) there exists $X \in \mathcal{C}$ such that $-e^{j} \bar{p}_{j}(B)+X \succeq-B$. Thus for any $m \in \mathcal{D}$ we have $\bar{p}_{j}(B) \leq \frac{m(B)}{m_{j}(\Omega)}$, implying $\bar{p}_{j}(B) \leq \underline{m}_{j}(B)$.

It is natural to ask under which condition we also have $\bar{p}_{j}(B) \geq \underline{m}_{j}(B)$, that would imply $\bar{p}_{j}(B)=\underline{m}_{j}(B)$ for bounded $B$. By Remark 3.3 we know that $\mathcal{Z}^{s} \subseteq \mathcal{D}$, hence it would be easy to get the desired inequality if

$$
\underline{m}_{j}(B)=\inf _{m \in \mathcal{D} \cap \operatorname{dom}\left(\mathbb{U}_{0}^{*}\right)} \frac{m(B)}{m_{j}(\Omega)}=\inf _{m \in \mathcal{D}} \frac{m(B)}{m_{j}(\Omega)} .
$$

This condition on $B$ (which looks hard to verify in practice) is, for example, automatically satisfied if the utility function $U$ is bounded from above (which implies that $0 \in \operatorname{dom}\left(\mathbb{U}_{0}^{*}\right)$, hence $\operatorname{dom}\left(\mathbb{U}_{0}^{*}\right)=\operatorname{ba}\left(\mathbb{R}_{+}^{D}\right)$ by Lemma 2.1 and Proposition 2.1).

Therefore if $B$ is in $L^{\infty}\left(\mathbb{R}_{+}^{D}\right)$ and $U$ is bounded from above, point (iii) of Proposition 4.2 tells us that the average price (in terms of any of the first $d$ assets) a risk averse agent is ready to pay to buy more and more units of a contingent claim and get always the same utility approaches a price that allows him to trade as to bear zero risk at maturity. If we only have boundedness of $B$, then, in general, the agent might keep some risk also in the limiting case.

REMARK 4.1 The definition of UIP can be further generalized to account for the case where we seek a "price" in terms of more than one asset. Let $n \leq d$ and denote $\bar{p}:=(p, 0) \in \mathbb{R}^{D}$ where $p \in \mathbb{R}^{n}$ and 0 is now the zero vector in $\mathbb{R}^{D-n}$. One can define $p(B) \in \mathbb{R}^{n}$, the UIP for $B$ expressed in terms of the first $n$ assets, as a solution to $u_{\mathcal{E}+B-\bar{p}}=u_{\mathcal{E}}$, with $\mathcal{E} \in \mathcal{O}$ and $\mathcal{E}+B-\bar{p} \in \mathcal{O}$. The subspace of $\mathbb{R}^{n}$ of the solutions to the previous inequality is closed if we only consider endowments in $L^{\infty}\left(\mathbb{R}^{D}\right.$ ) (by Lemma 4.1(iv)). A more thorough treatment of such vector UIP's is postponed to future research. 


\section{References}

[Bou02] B. Bouchard, Utility Maximization on the Real Line under Proportional Transaction Costs, Finance Stochast., 6 (2002), 495-516.

[BR06] C. Burgert and L. Rüschendorf, Consistent risk measures for portfolio vectors, Insurance: Mathematics and Economics, 38 (2006), 289-297.

[CO10] L. Campi and M. Owen, Multivariate Utility Maximization with Proportional Transaction Costs, to appear in Finance and Stochastics.

[CS06] L. Campi and W. Schachermayer, A super-replication theorem in Kabanov's model of transaction costs, Finance Stochast. 10 (2006), 579-596.

[CK96] J. Cvitanić and I. Karatzas. Hedging and portfolio optimization under transaction costs: a martingale approach, Mathematical Finance 6 (1996), 133-165.

[CSW01] J. Cvitanić, W. Schachermayer and H. Wang, Utility maximization in incomplete markets with random endowment, Finance Stochast., 5 (2001), 259-572.

[DN90] M. H. A. Davis and A. R. Norman, Portfolio selection with transaction costs, Math. Oper. Research 15 (1990), 676-713.

[DPT01] G. Deelstra, H. Pham and N. Touzi, Dual formulation of the utility maximization problem under transaction costs, Ann. Appl. Probab. 11 (2001), 1353-1383.

[DGR11] E. Denis, P. Guasoni and M. Rásonyi, The Fundamental Theorem of Asset Pricing under Transaction Costs. Preprint (2011).

[GK10] J. Grépat and Yu. M. Kabanov, Small Transaction Costs, Absence of Arbitrage and Consistent Price Systems, to appear in Finance and Stochastics.

[GRS10] P. Guasoni, M. Rásonyi and W. Schachermayer, The fundamental theorem of asset pricing for continuous processes under small transaction costs, Annals of Finance, 6 (2010), 157-191.

[HHR10] A. Hamel, F. Heyde and B. Rudloff, Set-valued risk measures for conical market models. Preprint (2010).

[HH09] V. Henderson and D. Hobson, Utility Indifference Pricing - An Overview, Chapter 2 in Indifference Pricing: Theory and Applications (ed. R. Carmona), Princeton University Press (2009).

[HN89] S. Hodges and A. Neuberger, Optimal replication of contingent claims under transaction costs, Review of Futures Markets, 8 (1989), 222-239.

[HG04] J. Hugonnier and D. Kramkov, Optimal investment with random endowments in incomplete markets, Annals of Applied Probability, 14 (2004), 845-864.

[JK95] E. Jouini and H. Kallal, Martingales and arbitrage in securities markets with transaction costs, J. Econ. Theory, 66 (1995), 178-197.

[JMT] E. Jouini, M. Meddeb and N. Touzi, Vector-valued coherent risk measures, Finance and Stochastics, 8 (2004), 531-552.

[Kab99] Yu. M. Kabanov, Hedging and liquidation under transaction costs in currency markets, Finance Stochast. 3 (1999), 237-248.

[KS09] Yu. M. Kabanov and M. Safarian, Markets with transaction costs, Springer Finance (2009).

[Kam01] K. Kamizono, Hedging and optimization under transaction costs. Ph.D. Thesis, Columbia University (2001).

[Kam04] K. Kamizono, Multivariate utility maximization under transaction costs. In: K. Akahori, O. Shigeyoshi and S. Watanabe (eds.) Stochastic Processes and Applications to Mathematical Finance: Proceedings of the Ritsumeikan International Symposium, pp. 133-149. World Scientific, (2004). 
[KrS99] D. Kramkov and W. Schachermayer, The asymptotic elasticity of utility functions and optimal investment in incomplete markets, Ann. Appl. Probab. 9 (1999), 904-950.

[Mu99] C. Munk, The valuation of contingent claims under portfolio constraints: reservation buying and selling prices. European Finance Review 3 (1999), 347-388.

[OZ09] M. P. Owen and G. Žitković, Optimal investment with an unbounded random endowment and utility-based pricing. Math. Finance 19 (2009), 129-159.

[RR83] K. P. S. B. Rao and M. B. Rao, Theory of charges: a study of finitely additive measures, Academic Press, London, 1983.

[Roc72] R. T. Rockafellar, Convex Analysis, Princeton University Press, Princeton, 1972.

[S04] W. Schachermayer, The Fundamental Theorem of Asset Pricing under Proportional Transaction Costs in Finite Discrete Time, Mathematical Finance 14 (2004), No. 1, 19-48.

[SS94] S. E. Shreve and H. M. Soner Optimal investment and consumption with transaction costs, Annals of Applied Probability 4 (1994), 609-692.

[Z02] C. Zălinescu Convex analysis in general vector spaces, World Scientific, New Jersey, 2002. 